\title{
THE THICKENING OF THE THIN DISK IN THE THIRD GALACTIC QUADRANT**
}

\author{
Giovanni Carraro $^{1,6}$, Rubén A. Vázquez ${ }^{2}$, Edgardo Costa ${ }^{3}$, Javier A. Ahumada $^{4}$, and Edgar E. Giorgi ${ }^{5}$ \\ ${ }_{1}^{1}$ European Southern Observatory, Alonso de Cordova 3107, Casilla 19001, Santiago 19, Chile; gcarraro@eso.org \\ ${ }^{2}$ Facultad de Ciencias Astronómicas y Geofisicas (UNLP), Instituto de Astrofísica de La Plata (CONICET, UNLP), \\ Paseo del Bosque s/n, La Plata, Argentina; rvazquez@fcaglp.unlp.edu.ar \\ ${ }^{3}$ Departamento de Astronomía, Universidad de Chile, Casilla 36-D, Santiago, Chile; costa@ das.uchile.cl \\ ${ }^{4}$ Observatorio Astronómico, Universidad Nacional de Córdoba, Laprida 854, 5000 Córdoba, Argentina; javier@oac.uncor.edu \\ ${ }^{5}$ Facultad de Ciencias Astronómicas y Geofísicas (UNLP), Instituto de Astrofísica de La Plata (CONICET, UNLP), \\ Paseo del Bosque s/n, La Plata, Argentina; egiorgi@fcaglp.unlp.edu.ar \\ Received 2014 March 28; accepted 2014 August 26; published 2014 December 10
}

\begin{abstract}
In the third Galactic quadrant $\left(180^{\circ} \leqslant l \leqslant 270^{\circ}\right)$ of the Milky Way, the Galactic thin disk exhibits a significant warp - shown both by gas and young stars - bending down a few kiloparsecs below the formal Galactic plane $\left(b=0^{\circ}\right)$. This warp shows its maximum at $l \sim 240^{\circ}$, in the direction of the Canis Major constellation. In a series of papers, we have traced the detailed structure of this region using open star clusters, putting particular emphasis on the spiral structure of the outer disk. We noted a conspicuous accumulation of young star clusters within $2-3 \mathrm{kpc}$ from the Sun and close to $b=0^{\circ}$, which we interpreted as the continuation of the Local (Orion) arm toward the outer disk. While most clusters (and young stars in their background) closely follow the warp of the disk, our decade-old survey of the spiral structure of this region led us to identify three clusters, Haffner 18 ( 1 and 2 ) and Haffner 19 , which remain very close to $b=0^{\circ}$ and lie at distances $(4.5, \sim 8.0$, and $6.4 \mathrm{kpc})$ where most of the material is already significantly warped. Here, we report on a search for clusters that share the same properties as Haffner 18 and 19, and investigate the possible reasons for such an unexpected occurrence. We present UBVRI photometry of five young clusters, namely NGC 2345, NGC 2374, Trumpler 9, Haffner 20, and Haffner 21, which also lie close to the formal Galactic plane. With the exception of Haffner 20, in the background of these clusters we detected young stars that appear close to $b=0^{\circ}$ and are located at distances up to $\sim 8 \mathrm{kpc}$ from the Sun, thus deviating significantly from the warp. These populations define a structure that distributes over almost the entire third Galactic quadrant. We discuss this structure in the context of a possible thin disk flaring, similar to the Galactic thick disk.
\end{abstract}

Key words: Galaxy: disk - Galaxy: structure - Hertzsprung-Russell and C-M diagrams - open clusters and associations: general - open clusters and associations: individual (Haffner 20, Haffner 21, NGC 2345, NGC 2374, Trumpler 9)

\section{INTRODUCTION}

The relevance of the study of open star clusters of the Milky Way is unique in many areas of modern astronomy, such as stellar astrophysics, stellar and Galactic evolution, and Galactic structure, and is widely recognized as such.

With this motivation, during the last decade we have been securing homogeneous $U B V R I_{k c}$ observations (same telescope, setup, and strategy of data analysis) of young open clusters in the third Galactic quadrant (3GQ: $180^{\circ} \leqslant l \leqslant 270^{\circ}$ ) of the Milky Way, in order to study its complicated structure. In this fashion, we have contributed significantly to the current understanding of the spiral structure in this Galactic region (Carraro et al. 2005; Moitinho et al. 2006; Vázquez et al. 2008; Carraro et al. 2010a).

Recent summaries of the status of this investigation and of the questions that still remain unanswered are given in Carraro (2014) and Carraro et al. (2014). Briefly, and using Figure 1 as a reference, our extensive campaign showed that the Local (Orion) arm (LOA) is an inter-arm feature, a bridge most likely connecting the Carina-Sagittarius arm in the first Galactic quadrant with the outer (Norma-Cygnus) arm in the 3GQ. The LOA also apparently breaks the Perseus arm, which is not

\footnotetext{
* Based on observations carried out at Cerro Tololo Inter-American Observatory, under programs CHILE-0008B-017 and CHILE-0010A-006.

6 On leave from Dipartimento di Astronomia, Università di Padova, Italy.
}

prominent at all in young stars or gas in this region of the Galaxy. The extension of the LOA into the 3GQ occurs toward $l \sim 240^{\circ}$, that is, in the direction of the Canis Major overdensity $\left(b \sim-8^{\circ}\right.$, Martin et al. 2004; Momany et al. 2006; Moitinho et al. 2006). This picture confirms independent findings based on $\mathrm{H}_{\mathrm{I}}$ studies in the same region (Levine et al. 2006). Because our sample extends up to $20 \mathrm{kpc}$ from the Galactic center, we were also able to trace the progressive bending of the thin disk caused by the warp, and did not find any indication of a disk cutoff or truncation at $14 \mathrm{kpc}$ from the Galactic center, as previously postulated using Galactic models (Robin et al. 1992).

Two of the main open questions that arise from Figure 1 are (1) at what distance from the Sun the disk starts to bend down and (2) whether flaring (the thickening of the disk in the vertical direction), clearly visible in the old disk population (Momany et al. 2006; López-Corredoira \& Molgó 2014), has some impact on the structure of the Galactic thin disk as well.

Our previous studies indicate that the warp becomes important beyond $12 \mathrm{kpc}$ from the Galactic center $(\sim 4 \mathrm{kpc}$ from the Sun). From the works of Vázquez et al. (2008, Figure 6), and Vázquez et al. (2010, Figure 15), it is evident that the disk stays close to the formal $\left(b=0^{\circ}\right)$ plane up to about $12 \mathrm{kpc}$, and that it is very thin $( \pm 100 \mathrm{pc})$. Beyond this distance, the thin disk starts bending, and at the same time its vertical extent gets more and more significant. Although the 


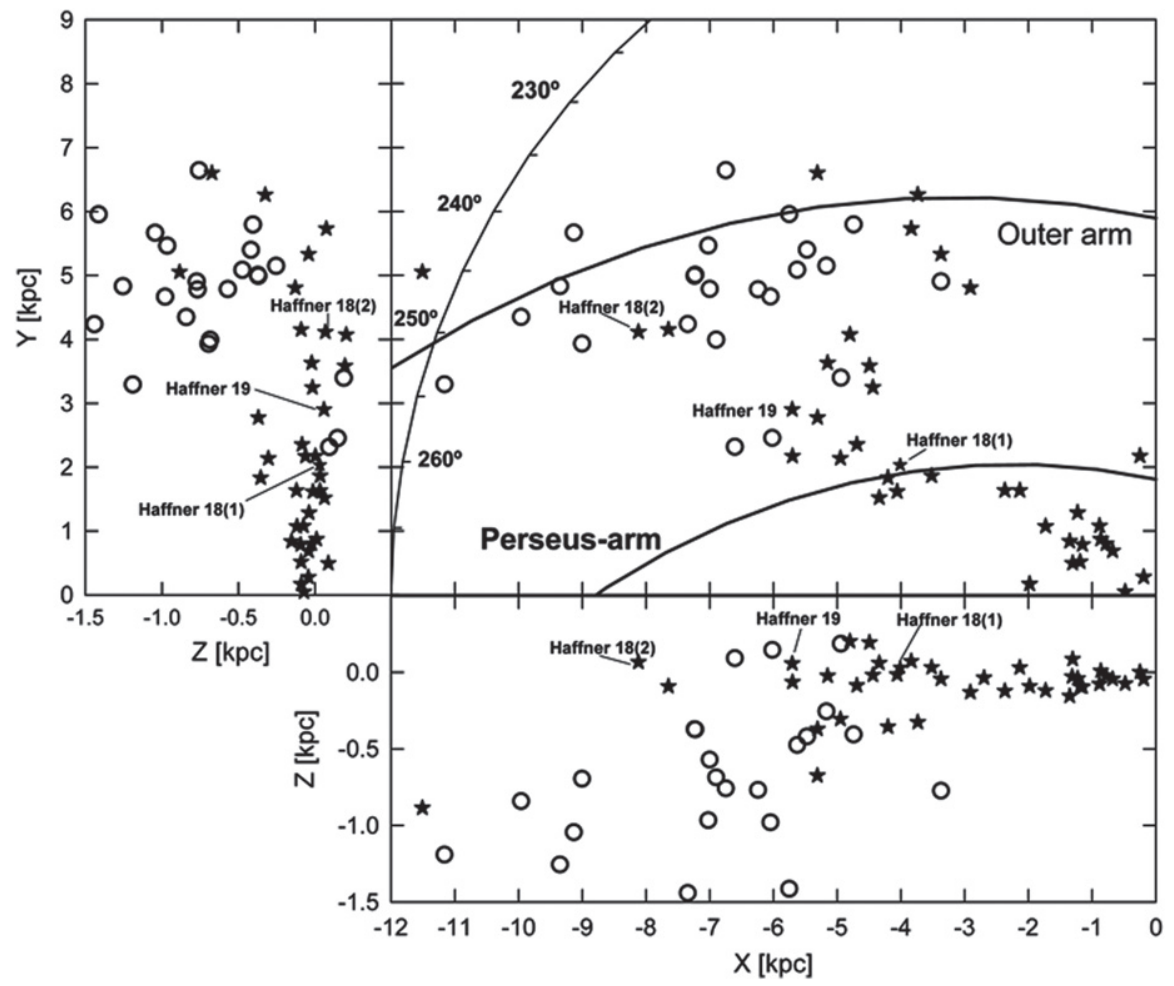

Figure 1. Thin-disk structure in the third Galactic quadrant as traced by young open clusters and background populations. The large panel shows the plane of the disk, while the two smaller panels show the $X-Z$ and $Y-Z$ projections. Note the particular location of the star clusters Haffner $18(1 / 2)$ and 19 , above the formal $\left(b=0^{\circ}\right)$ plane of the Galaxy. Symbols are as follows: stars are young open clusters, and empty circles are background populations of early-type stars.

Table 1

Cluster Coordinates for $\mathbf{J} 2000.0$

\begin{tabular}{lcccr}
\hline \hline Cluster & R.A. & Decl. & $l$ & \multicolumn{1}{c}{$b$} \\
\hline NGC 2345 & $07: 08: 18$ & $-13: 11: 36$ & 226.58 & -2.31 \\
NGC 2374 & $07: 23: 56$ & $-13: 15: 48$ & 228.41 & 1.02 \\
Trumpler 9 & $07: 55: 40$ & $-25: 53: 00$ & 243.07 & 1.28 \\
Haffner 21 & $08: 01: 09$ & $-27: 13: 00$ & 244.85 & 1.63 \\
Haffner 20 & $07: 56: 15$ & $-30: 22: 00$ & 246.97 & -0.93 \\
\hline
\end{tabular}

bulk of young star clusters follows the warp closely, there is a group of young star clusters, like Haffner 18(1/2) and Haffner 19 (at 4.5, 8, and $6.4 \mathrm{kpc}$; see Figure 1), that clearly and unexpectedly deviate from the main disk locus. Although these clusters are relatively nearby, we found young stellar populations in their background at distances up to $9 \mathrm{kpc}$ from the Sun that also do not follow the warp. These latter might indicate a flaring of the thin disk or, more conservatively, they could be tracing local irregularities (e.g., disk corrugations) of the same kind as the Big Dent (Alfaro et al. 1991; Moitinho et al. 2006)

In this paper, we report on an extensive search for other presumably young clusters in the same Galactic sector, and close to the formal Galactic plane, like Haffner 18 and 19. The aim of this study is twofold; we want (1) to improve the basic parameters of a set of poorly studied but potentially interesting clusters, and (2) to use them to consolidate our knowledge of the thin disk structure in the 3GQ. Specifically, we present results for five open clusters (see Table 1): NGC 2345 (C0706 -130), NGC 2374 (C0721-131), Haffner 20 (C0754-302), Haffner 21 (C0759-270), and Trumpler 9 (C0753-258), based on new $U B V R I_{k c}$ data.

Since these five clusters have been very poorly studied, a significant part of this paper is dedicated to the description of the data and the derivation of their properties. Therefore, the outline of the paper is as follows. In Section 2, we present a brief literature overview of the five clusters; in Section 3, we present the observations, the data reduction procedure, and the photometry; in Section 4, we make a comparison of our data set with previous investigations; in Section 5, we describe the star count procedure and derivation of the cluster sizes; and in Section 6, we present the analysis of the clusters' photometric diagrams. In Section 7, we discuss the properties and spatial distribution of early-type field stars in the clusters back- and foreground. Finally, in Section 8, we summarize the main conclusions of our work.

\section{LITERATURE OVERVIEW}

A search of the literature shows that data for the five clusters are truly scarce, and that no modern (CCD) studies are available.

$N G C 2345$. NGC 2345 is an open cluster projected toward the Monoceros constellation. Moffat (1974) carried out photoelectric photometry of 62 stars down to $V=14$. He detected variable reddening $\left(E_{(B-V)}=0.5\right.$ to $\left.\sim 1.10\right)$, and placed the cluster at a distance of $1.75 \mathrm{kpc}$. Using star counts, Moffat found that the cluster diameter is 10.5 arcmin, but indicated that, using only faint stars, it could be as large as 12.3 arcmin. Interesting to this study, Moffat also isolated a small number of blue stars that he classified as OB using objective prism observations, and that could be at distances as large as $10 \mathrm{kpc}$. Lastly, from the inspection of photometric diagrams, he inferred an age of $\sim 6 \times 10^{6}$ year for the cluster.

NGC 2374. Like NGC 2345, this cluster is projected toward the Monoceros constellation. Photographic and photoelectric photometry for 39 stars was secured by Babu (1985), who estimated a uniform color excess $E(B-V)=0.175$, an age of 
Table 2

Log of Observations

\begin{tabular}{|c|c|c|c|c|c|}
\hline Date & Cluster & Filter & Exposure Time (sec) & Airmass & $\overline{\text { Seeing }}$ \\
\hline \multirow[t]{8}{*}{2010 Dec 30} & Haffner 20 & $V$ & $2 \times 10,60,900$ & $1.00-1.01$ & $0.9-1.2$ \\
\hline & & $B$ & $20,3 \times 150$ & $1.04-1.06$ & $0.9-1.2$ \\
\hline & & $U$ & 30,300 & $1.06-1.07$ & $0.9-1.2$ \\
\hline & & $I$ & $3 \times 10,60,900$ & $1.02-1.03$ & $0.9-1.2$ \\
\hline & Trumpler 9 & V & $2 \times 10,60,900$ & 1.00 & $0.9-1.2$ \\
\hline & & $B$ & $20,150,1500$ & $1.12-1.19$ & $0.9-1.2$ \\
\hline & & $U$ & $2 \times 30,300,2000$ & $1.00-1.03$ & $0.9-1.2$ \\
\hline & & $I$ & $2 \times 10,60,900$ & $1.20-1.28$ & $0.9-1.2$ \\
\hline \multirow[t]{6}{*}{2010 Dec 31} & Haffner 20 & $U$ & $2 \times 20,2000$ & $1.25-1.27$ & $0.8-1.2$ \\
\hline & & $B$ & 20,1500 & $1.37-1.38$ & $0.8-1.2$ \\
\hline & Haffner 21 & $U$ & $30,300,2000$ & $1.12-1.14$ & $0.8-1.2$ \\
\hline & & $B$ & $20,150,1500$ & $1.02-1.04$ & $0.8-1.2$ \\
\hline & & V & $10,60,900$ & 1.00 & $0.8-1.2$ \\
\hline & & $I$ & $2 \times 10,60,900$ & $1.00-1.01$ & $0.8-1.2$ \\
\hline \multirow[t]{10}{*}{2008 Feb 01} & NGC 2345 & $U$ & $5,20,100,200$ & $1.09-1.10$ & $1.0-1.4$ \\
\hline & & $B$ & $5,20,100,200$ & $1.07-1.08$ & $1.0-1.4$ \\
\hline & & V & $5,10,60,120$ & $1.08-1.09$ & $1.0-1.4$ \\
\hline & & $R$ & $5,10,60,120$ & $1.09-1.12$ & $1.0-1.4$ \\
\hline & & $I$ & $5,10,100,200$ & $1.06-1.07$ & $1.0-1.4$ \\
\hline & NGC 2374 & $U$ & $5,20,100,200$ & $1.11-1.12$ & $1.0-1.4$ \\
\hline & & $B$ & $5,20,100,200$ & $1.14-1.15$ & $1.0-1.4$ \\
\hline & & V & $5,10,60,120$ & $1.22-1.23$ & $1.0-1.4$ \\
\hline & & $R$ & $5,10,60,120$ & $1.25-1.27$ & $1.0-1.4$ \\
\hline & & $I$ & $5,10,100,200$ & $1.17-1.19$ & $1.0-1.4$ \\
\hline \multirow[t]{10}{*}{2008 Feb 03} & NGC 2345 & $U$ & $60,600,1500$ & $1.05-1.07$ & $0.9-1.1$ \\
\hline & & $B$ & $30,600,1500$ & $1.05-1.08$ & $0.9-1.1$ \\
\hline & & V & $30,600,1200$ & $1.08-1.09$ & $0.9-1.1$ \\
\hline & & $R$ & $30,600,1200$ & $1.09-1.12$ & $0.9-1.1$ \\
\hline & & $I$ & $30,600,1200$ & $1.07-1.08$ & $0.9-1.1$ \\
\hline & NGC 2374 & $U$ & $60,600,1500$ & $1.06-1.07$ & $0.9-1.1$ \\
\hline & & $B$ & $30,600,1500$ & $1.11-1.13$ & $0.9-1.1$ \\
\hline & & V & $30,600,1200$ & $1.31-1.38$ & $0.9-1.1$ \\
\hline & & $R$ & $30,600,1200$ & $1.50-1.60$ & $0.9-1.1$ \\
\hline & & $I$ & $30,600,1200$ & $1.19-1.24$ & $0.9-1.1$ \\
\hline
\end{tabular}

$75 \times 10^{6}$ year, and a distance of $1.2 \mathrm{kpc}$. We note that the age derived by Babu (1985) is significantly younger than previous estimates, such as those from Fenkart et al. (1972) and Lyngå (1980), who report ages of $3.5 \times 10^{8}$ and $2 \times 10^{9}$ year, respectively.

Trumpler 9. Trumpler 9 is a Puppis association object. Pişmiş (1970), using photoelectric and photographic data, found that the earliest spectral type is $\mathrm{A} 0$, that its reddening in $(B-V)$ is at most $0.2 \mathrm{mag}$, and that its spectroscopic distance is approximately 900 pc. Vogt \& Moffat (1972) used photoelectric $U B V-\mathrm{H} \beta$ photometry and derived a larger reddening $E(B-V)=0.29$, an apparent distance modulus of 11.98 , and a distance of $1.62 \mathrm{kpc}$. Their estimated bluest spectral type is B1, very different from Pişmiş's results.

Haffner 21 and 20. These two clusters also belong to the Puppis association. Based on photographic photometry, Fitzgerald \& Moffat (1974) established B9 as the earliest spectral type present in Haffner 20 and Haffner 21. They also determined a distance and reddening of $2.4 \mathrm{kpc}$ and $0.55 \mathrm{mag}$ for Haffner 20, and of $3.3 \mathrm{kpc}$ and $0.20 \mathrm{mag}$ for Haffner 21, respectively.

\section{OBSERVATIONS, DATA REDUCTION, AND PHOTOMETRY}

In this section, we briefly introduce the observation strategy, the collected data, and the technique employed to reduce them.
Since this work is part of a long series of papers, full details about the observations, data reduction procedure, and the photometry can be found in previous papers from our group (see, e.g., Carraro et al. 2010a, 2010b).

\subsection{Observations}

Observations were carried out with the Y4KCAM camera attached to the Cerro Tololo Inter-American Observatory (CTIO, Chile) $1 \mathrm{~m}$ telescope, operated by the SMARTS consortium $^{7}$, in 2008 February and 2010 December. This camera is equipped with an STA $4064 \times 4064 \mathrm{CCD}^{8}$ with $15 \mu \mathrm{m}$ pixels, yielding a scale of $0^{\prime} .289$ pixel $^{-1}$ and a field of view of $20^{\prime} \times 20^{\prime}$ at the Cassegrain focus of the telescope.

In Table 2, we present the log of our UBVRI observations. All observations were carried out in photometric, good-seeing conditions. Our UBVRI instrumental photometric system was defined using a standard broadband Kitt Peak $U B V R I_{k c}$ set of filters. ${ }^{9}$ To determine the transformation from our instrumental system to the standard Johnson-Kron-Cousins system, and to correct for extinction, each night we observed Landolt's area SA 98 (Landolt 1992) multiple times (three or four), with different air masses ranging from $\sim 1.1$ to $\sim 2.2$. Field SA 98

\footnotetext{
7 www.astro.yale.edu/smarts

8 www.astronomy.ohio-state.edu/Y4KCam/detector.html

9 www.astronomy.ohio-state.edu/Y4KCam/filters.html
} 
Table 3

Coefficients of the Transformation Equations

\begin{tabular}{|c|c|c|c|c|c|c|}
\hline Mag & $c_{0}$ & $\sigma$ & $c_{1}$ & $\sigma$ & $c_{2}$ & $\sigma$ \\
\hline \multicolumn{7}{|c|}{ CTIO, $1 \mathrm{~m}$ telescope, 2008 February 01} \\
\hline$U$ & +1.35 & 0.01 & -0.49 & 0.01 & +0.015 & 0.016 \\
\hline$B$ & +2.27 & 0.01 & -0.25 & 0.01 & -0.100 & 0.015 \\
\hline$V$ & +2.36 & 0.01 & -0.17 & 0.01 & -0.010 & 0.005 \\
\hline$R$ & +2.10 & 0.01 & -0.10 & 0.01 & 0.048 & 0.005 \\
\hline$I$ & +1.36 & 0.01 & -0.07 & 0.01 & -0.043 & 0.005 \\
\hline \multicolumn{7}{|c|}{ CTIO, $1 \mathrm{~m}$ telescope, 2008 February 03} \\
\hline$U$ & +1.35 & 0.01 & -0.49 & 0.01 & +0.014 & 0.020 \\
\hline$B$ & +2.20 & 0.02 & -0.25 & 0.01 & -0.097 & 0.014 \\
\hline$V$ & +2.32 & 0.01 & -0.16 & 0.01 & -0.012 & 0.006 \\
\hline$R$ & +2.14 & 0.01 & -0.09 & 0.01 & 0.050 & 0.010 \\
\hline$I$ & +1.35 & 0.01 & -0.08 & 0.01 & -0.045 & 0.005 \\
\hline \multicolumn{7}{|c|}{ CTIO, $1 \mathrm{~m}$ telescope, 2010 December 30} \\
\hline$U$ & +0.89 & 0.02 & -0.52 & 0.01 & +0.019 & 0.008 \\
\hline$B$ & +2.09 & 0.02 & -0.27 & 0.01 & -0.115 & 0.007 \\
\hline$V$ & +2.30 & 0.01 & -0.15 & 0.01 & +0.016 & 0.005 \\
\hline$I$ & +1.29 & 0.01 & -0.06 & 0.01 & -0.045 & 0.005 \\
\hline \multicolumn{7}{|c|}{ CTIO, 1 m telescope, 2010 December 31} \\
\hline$U$ & +0.90 & 0.01 & -0.50 & 0.01 & +0.032 & 0.006 \\
\hline$B$ & +2.10 & 0.01 & -0.25 & 0.01 & -0.112 & 0.005 \\
\hline$V$ & +2.26 & 0.01 & -0.11 & 0.01 & +0.030 & 0.005 \\
\hline$I$ & +1.30 & 0.01 & -0.07 & 0.01 & -0.048 & 0.005 \\
\hline
\end{tabular}

includes over 40 well-observed standard stars with good magnitude and color coverage: $9.5 \leqslant V \leqslant 15.8$, $-0.2 \leqslant(B-V) \leqslant 2.2,-0.3 \leqslant(U-B) \leqslant 2.1$.

\subsection{Reductions}

A basic reduction of the CCD frames was performed using the Yale/SMARTS $y 4 \mathrm{k}$ reduction script based on the IRAF ${ }^{10}$ package CCDRED, which includes bias and sky-flat corrections. Photometry was then performed using IRAF's DAOPHOT and PHOTCAL packages. Instrumental magnitudes were extracted following the point-spread function (PSF) method (Stetson 1987) using a quadratic, spatially variable master PSF (PENNY function). We typically use a minimum of $50 \mathrm{PSF}$ stars spread over the detector. Finally, the PSF photometry was aperture-corrected using aperture corrections determined by making aperture photometry of bright, isolated stars in the field.

\subsection{The Photometry}

To transform our instrumental magnitudes to the standard system, we used equations of the form

$$
M=m+c_{0}+c_{1} \times X+c_{2} \times(B-V),
$$

where $M$ and $m$ are the standard and instrumental magnitudes, $X$ is the airmass, and $c_{0}, c_{1}$, and $c_{2}$ are the zero point, first-order extinction, and color term coefficients, respectively. We note that the use of second-order extinction terms did not improve the fit. In Table 3, we list the coefficients of the transformation equations and their corresponding errors for each night.

In this way, we produced calibrated $U B V R I_{\mathrm{kc}}$ photometric catalogs for NGC 2345, NGC 2374, Haffner 20, Haffner 21, and Trumpler 9, containing 6326, 3266, 3740, 6725, and 6119 entries, respectively.

\footnotetext{
${ }^{10}$ IRAF is distributed by the National Optical Astronomy Observatory, which is operated by the Association of Universities for Research in Astronomy, Inc., under cooperative agreement with the National Science Foundation.
}

These optical catalogs were cross-correlated with 2MASS (Skrutskie et al. 2006) to convert pixel (i.e., detectorcoordinated) into equatorial R.A. and decl. for equinox J2000.0, thus providing 2MASS-based astrometry.

\section{COMPARISON WITH PREVIOUS STUDIES}

As mentioned previously, no modern CCD studies exist for the five clusters we are presenting in this study, so we want to note that we are making a comparison of our CCD data only with photographic and photoelectric data. In general, photoelectric data are precise, but suffer from two major problems: they are not very deep, and in crowded fields they cannot resolve blends because of the relatively large fixed apertures adopted. On the other hand, photographic data are in general somewhat deeper, but are less precise, and quickly lose efficiency near the sensitivity limit because of poor quantum efficiency.

Figures 2 and 3 show the results of the comparison; below, we comment on a cluster-by-cluster basis. We note that all mean differences presented were computed in the sense of our measurements minus others, and errors on the mean are standard deviations.

NGC 2345. Photometric differences with photoelectric measures for 62 stars in common with Moffat (1974) are shown in the upper panels of Figure 2. Mean differences are $\Delta V=-0.086 \pm 0.07, \quad \Delta(B-V)=-0.015 \pm 0.03, \quad$ and $\Delta(U-B)=+0.04 \pm 0.08$. While the colors are in fine agreement, our $V$ magnitudes are somewhat brighter, but still comparable within the errors. Moffat explains that his observations were carried out using a small telescope. Therefore, we believe this may lead to probable contamination in the star light due to the presence of faint neighbors in the diaphragm area and/or difficulties in removing the sky contribution in the star light. These are the typical limitations associated with photoelectric photometry. In this respect, CCD imaging combined with PSF photometry allows a more precise removal of the contamination sources and also better isolation of the star light.

NGC 2374. Photometric differences for 36 stars in common with Babu (1985) are shown in the lower panels of Figure 2. If we take all 36 stars into account (photoelectric and photographic data taken together), the mean differences are $\Delta V=-0.16 \pm 0.16, \quad \Delta(B-V)=+0.108 \pm 0.23, \quad$ and $\Delta(U-B)=+0.11 \pm 0.22$. Excluding the 10 stars with photographic photometry (all fainter than $V \sim 14.0$ ), the mean differences become $\Delta V=-0.15 \pm 0.09, \Delta(B-V)=$ $-0.01 \pm 0.05$, and $\Delta(U-B)=+0.03 \pm 011$. In this last case, the scatter around the means significantly decreases and the color agreement is fine. However, our $V$ magnitudes still appear slightly brighter with a large scatter. We could not find any detailed information about either the quality of the photoelectric observing runs by Babu (1985; made at the Kevalur $102 \mathrm{~cm}$ telescope in India) or related to the errors involved in the measurements, but we draw the attention to Babu, who suggests photographic mean errors above 0.2 for $B$ and $V$ and even larger for $U$. This said, one can understand the zero-point offset between the Babu photoelectric magnitudes and ours.

Trumpler 9. The photometric comparison is shown in the upper panels of Figure 3. Empty triangles depict the comparison with the photographic data from Pişmiş (1970), while the comparison to her photoelectric data is depicted by 


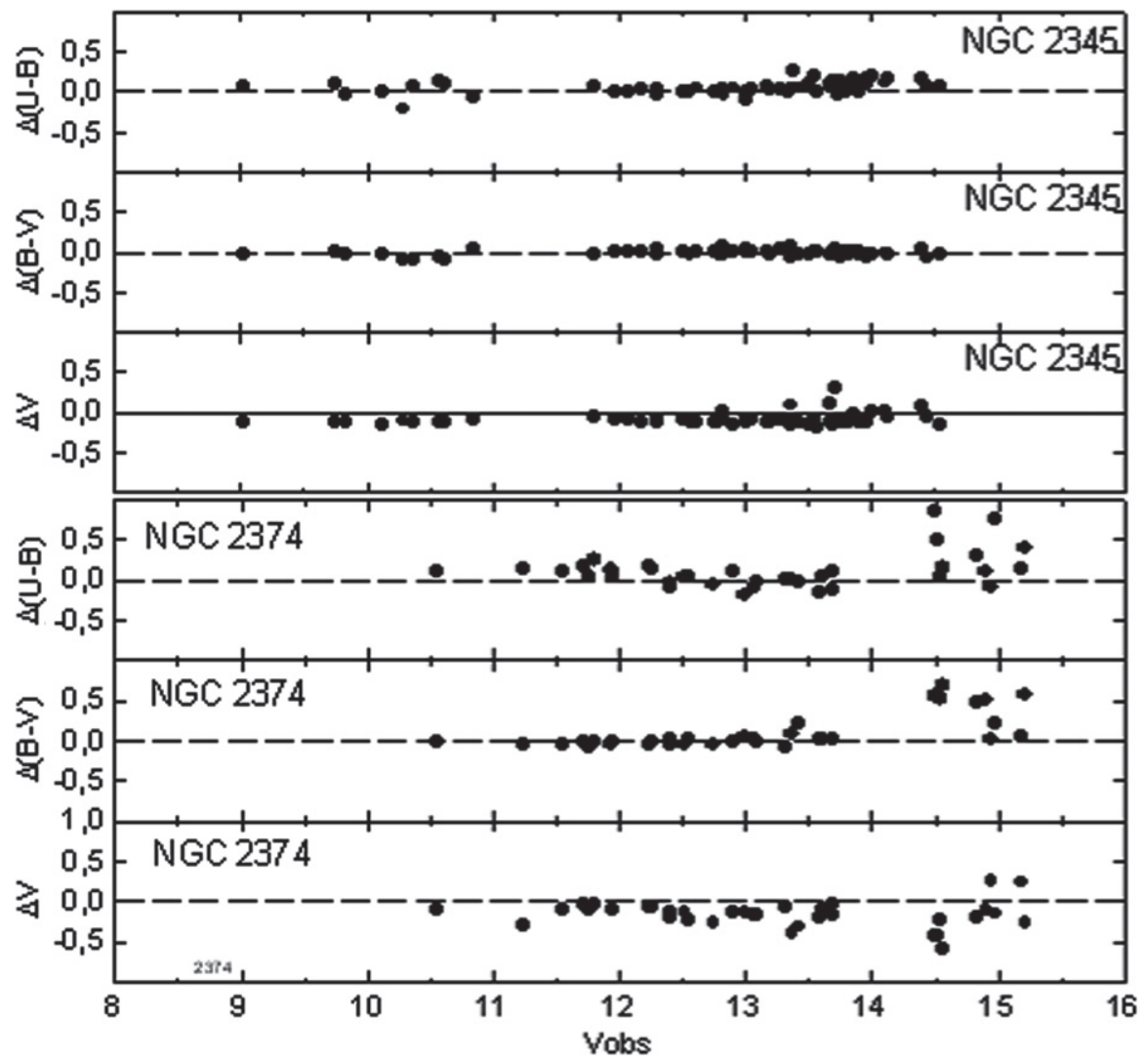

Figure 2. Comparison of our CCD photometry with literature sources for NGC 2345 and NGC 2374. Differences are in the sense of our measurements minus published data. See the text for details.

filled circles. Filled squares show the comparison against the photoelectric photometry by Vogt \& Moffat (1972). The comparison with photographic photometry from Pişmiş (1970) yielded $\Delta V=+0.17 \pm 0.08, \Delta(B-V)=-0.09 \pm 0.13$, and $\Delta(U-B)=-0.06 \pm 0.18$ for 13 stars in common. Both the offset in $V$ and the scatter around the mean are strong. The comparison is worse with photoelectric measures for 10 other stars from Pişmiş (1970). In this case, we found the following differences: $\Delta V=-0.50 \pm 0.64, \Delta(B-V)=0.01 \pm 0.08$, and $\Delta(U-B)=0.01 \pm 0.12$. The mean $\Delta V$ is absolutely unrealistic. Such a curious mean difference leads us to conclude that some of her photoelectric $V$ measures are simply wrong. There is also, in our opinion, a propagation effect producing the large $V$ offset with photographic and photoelectric photometry since they come from a bad data calibration by Pişmiş (1970). In brief, she used the wrong photoelectric sequence to put her photographic data into the standard system. This scenario is supported by a comparison with Vogt \& Moffat (1972) photometry. We found 20 stars in common with their photoelectric measures. As seen in Figure 3, there is a significant discrepancy in $V$ for six of them. Once these these six problematic stars have been removed, the mean differences with Vogt \& Moffat (1972) are $\Delta V=+0.05 \pm 0.06$, $\Delta(B-V)=0.00 \pm 0.04$, and $\Delta(U-B)=0.03 \pm 0.12$. The $V$ offset is relatively small and well within the error. The six deviating stars are either misidentified or variable objects.

Haffner 20 and 21. A comparison with the photographic photometry of Fitzgerald \& Moffat (1974) is shown in Figure 3; the middle panels are Haffner 21 (47 stars), and in the bottom panels are Haffner 20 (31 stars). Mean differences are $\Delta V=-0.09 \pm 0.08, \Delta(B-V)=+0.05 \pm 0.09$, and $\Delta(U-B)=+0.03 \pm 0.15$ for Haffner 21. For Haffner 20, they are $\Delta V=+0.06 \pm 0.09, \Delta(B-V)=+0.06 \pm 0.11$, and $\Delta(U-B)=-0.13 \pm 0.14$. In both clusters, most of the scatter comes from the faint tail of the photometry, as one can readily see inspecting Figure 3. However, and particularly in the case of Haffner 20, there is a distinctive group of largely deviating stars, some of them for more than $0.8 \mathrm{mag}$ in $V$. These stars are distributed over the whole magnitude range, which makes it impossible to separate images of close stars in the photographic plates and obtain their true photometric values. It must also be noted that the calibration of photographic magnitudes by Fitzgerald \& Moffat (1974) was performed using (secondary) photoelectric standards from some nearby open cluster sequences. Fitzgerald \& Moffat (1974) mention that the external errors of their calibration are of the order of 0.09 in $V, B$, and $U$ filters. As for the internal standard errors of the mean, they claim they are all above 0.05 in $V, B-V$, and $U-B$. This large uncertainty in the old photographic measures can easily explain the differences with our photometry.

\section{STAR COUNTS AND CLUSTER SIZE}

Linear size is a fundamental quantity in comprehensively characterizing a star cluster. It is necessary to estimate its evolutionary status through an analysis of its density profile and other related parameters (see Aarseth et al. 1996; de La Fuente Marcos 1997; Kroupa et al. 2001) as the slope of the mass function. It is also routinely used as a criterion for 


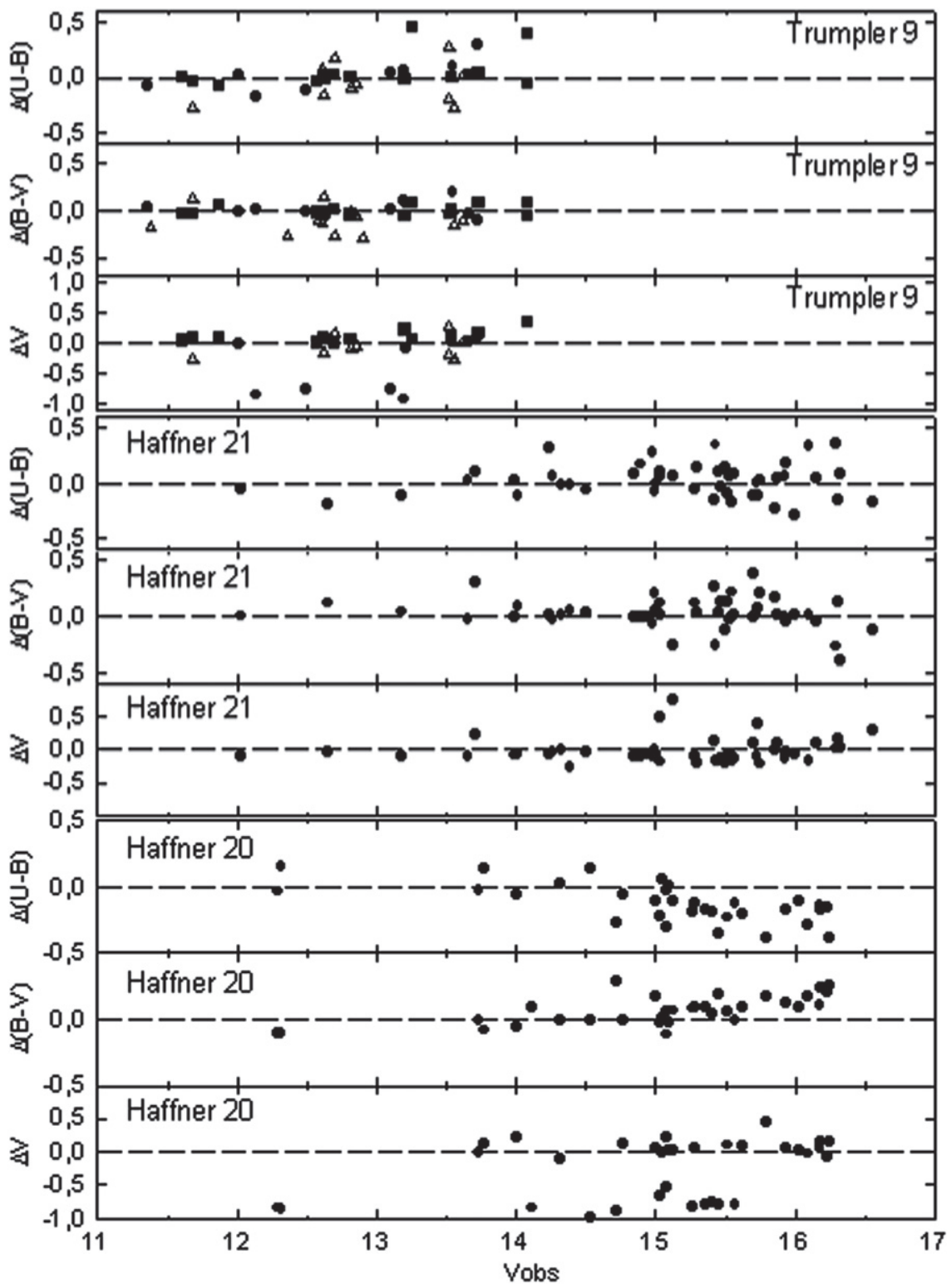

Figure 3. Comparison of our CCD photometry for Trumpler 9, Haffner 20, and Haffner 21. Differences are in the sense of our measurements minus published data. See the text for symbols in Trumpler 9.

separating cluster-dominated from field-dominated regions in photometric diagrams (Baume et al. 2004).

The usual way to estimate the radius of a star clusters is to assume a centrally peaked spherical stellar density distribution and determine the distance at which the density converges to the mean field background. This determination is done either by visually setting the limit in a plot or by fitting King (1962) or other profiles to the run of radial density. However, both approaches assume a spherically symmetric distribution and, in the case of the King profiles, it also assumes that the system is dynamically relaxed. This is not at all the case for Galactic open clusters.

Since we are mostly interested in determining the cluster radii to reduce field contamination in photometric diagrams and derive the best possible cluster parameters, the following approach has therefore been adopted. Contour maps were computed in each field by choosing adequate kernel sizes and grid steps. Once a notorious stellar density is identified, a circle enclosing its full range is drawn by eye and the cluster limit is taken as that defined by the radius of this circle. We adopt the cluster center to be the geometric center of the cluster, as opposed to the position of the density peak. We want to emphasize that unlike what usually happens with globular clusters, the center of an open cluster can rarely be unambiguously identified. Furthermore, assigning a unique center value to an open cluster with complicated non-circular morphology or one that is poorly populated may often carry unclear implications of what precisely that center represents, in terms of the cluster's structure.

Figure 4 shows contour maps of the five clusters under analysis. In the inset in each figure, the color coding represents the number of stars per $\operatorname{arcmin}^{2}$. With the exception of NGC 2374, we detect in all cases a clear overdensity around the nominal cluster coordinates. As for NGC 2374, if the cluster exists, it might simply be larger than the area we covered with our photometry since no clear overdensity is appreciable in 

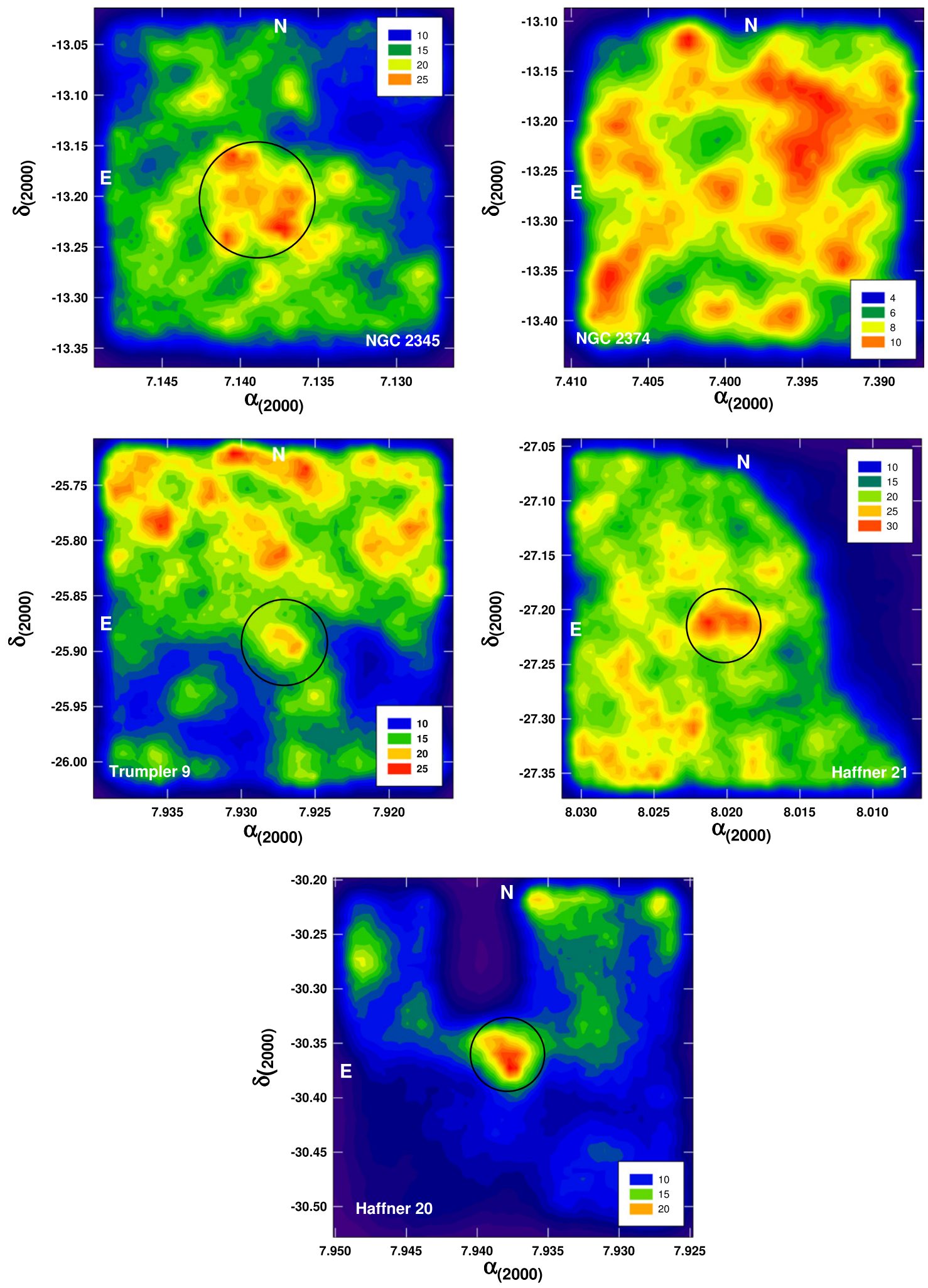

Figure 4. Iso-density contour maps for the five clusters studied.

Figure 4. NGC 2345 and Haffner 21 emerge clearly as star clusters, but their regions suffer from variable extinction exemplified by the conspicuous dust lanes, in both cases in the northwest area of the corresponding images. The most evident peak is that of Haffner 20, while Trumpler 9 shows several peaks, mostly in the northern region. The central peak in
Trumpler 9, however, is evident enough to consider it a star cluster since it coincides with the cluster location in earlier studies, and we use it to set its limits. We remind readers that our method is intended to yield only an approximation of the cluster size because of the loose and asymmetric nature of open star clusters and the often wrong impression of an overdensity 


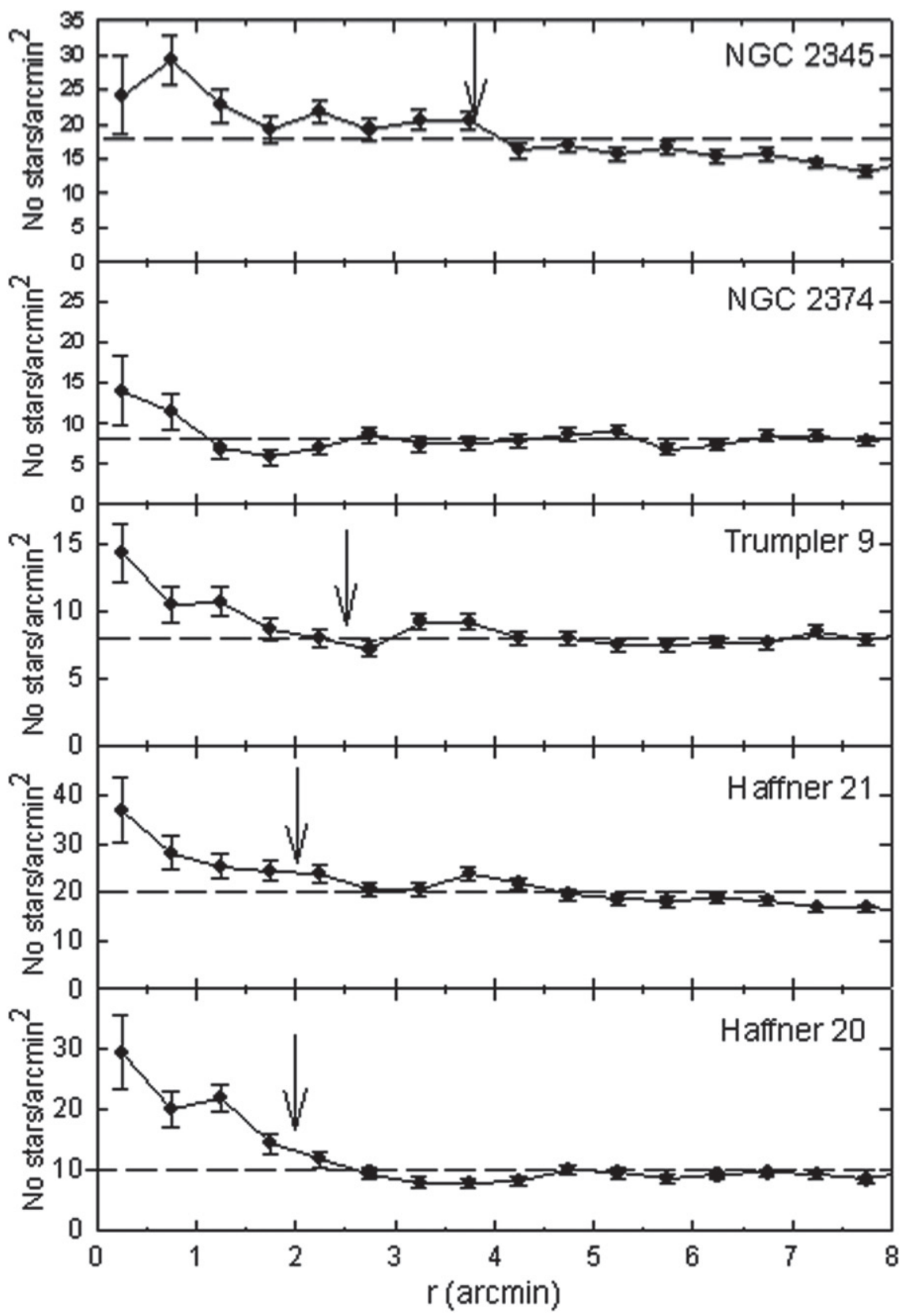

Figure 5. Star counts as a function of radius. See the text for details.

induced by variable visual absorption. This is particularly important for Trumpler 9, Haffner 21, and NGC 2345, where the variable extinction in the stellar field introduces important fluctuations in the star density background, which, in turn, alters the cluster extension.

We then used star counts to derive the radial density profile and measure the cluster radial extent, improving over previous, mostly visual, estimates. The results are shown in Figure 5. In all cases, a distinct overdensity is detected, with radii of $\sim 4.0$, 2.5, 4.0, 2.0, and 1.0 arcmin for NGC 2345, NGC 2374, Trumpler 9, Haffner 21, and Haffner 20 (from top to bottom in Figure 5), respectively. In the case of NGC 2374, the radius of 1.4 arcmin refers to the central overdensity seen in Figure 4 and commented on in Section 6.

\section{ANALYSIS OF THE CLUSTERS' PHOTOMETRIC DIAGRAMS}

To derive the basic parameters of the star clusters under study, we have performed a detailed inspection of the two-color diagram (TCD) and color-magnitude diagram (CMD) after having verified their reliability and defined the angular cluster sizes according to the results found in Section 5. The next steps in the analysis are to identify the cluster sequences in all of the photometric diagrams, determine the color excesses by shifting the Schmidt-Kaler (1982) to fit the sequences in the TCD, and estimate the distance by superposition of the ZAMS onto the blue envelope of the corresponding star sequence in the CMD. In each case, both the fit and its error have been estimated by eye on a trial and error basis. Once the mean reddening and 

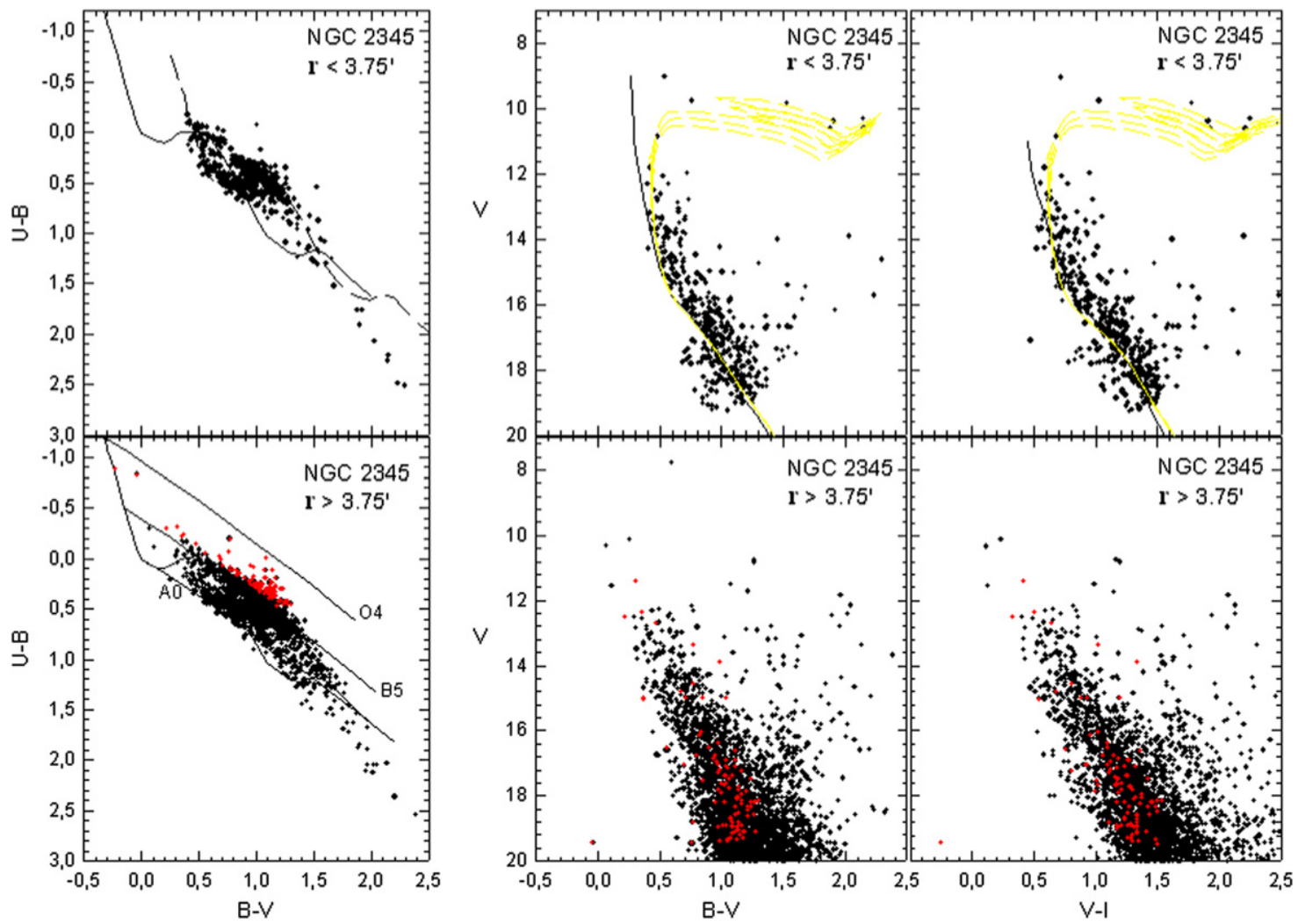

Figure 6. Color-color and color-magnitude diagrams for NGC 2345 (upper panels), and for the surrounding field (lower panels). Dashed lines are isochrones from Marigo et al. (2008) shifted by color excess and distance modulus. The solid line in the two-color diagram is a reddening-free ZAMS, while the dashed line is the same ZAMS shifted by the color excess. Two reference spectral types are indicated in the lower left panel, together with the reddening vector. See the text for details.

distance have been determined, we fit isochrones (Marigo et al. 2008) computed with solar metallicity. Again, this fitting has been performed by eye, but instead of reporting the error in the fitting we suggest a range of values. As for the value of the absorption law (the R-value) in each cluster, we have adopted $A_{V}=R \times E_{(B-V)}$ with $R=3.1$. Our adoption of this $R$ value is supported in a long series of articles; see, for instance, Moitinho (2001), Carraro et al. (2007), or Vázquez et al. (2010). In fact, we have demonstrated, using the B-V, V-I color-color diagrams, that in the quadrant of the Galaxy the absorption law is normal with very few exceptions, as Haffer 18/19 discussed in Vázquez et al. (2010). Finally, we reiterate once again that our methodology is a classical and well-known procedure (see Straizys 1991 for an exhaustive review). An error analysis has been conducted following the Carraro et al. (2007) and Carraro (2011) propagation formulae, which take into account the effects of spectral misclassification on distance and reddening.

In the following we only comment on the results, on a cluster-by-cluster basis.

NGC 2345. The TCD and CMDs shown in the upper panels of Figure 6 were constructed using all stars within 3.75 arcmin from the cluster center. The TCD shows a clear and slightly blue sequence partially contaminated by field interlopers as seen in the corresponding CMDs. Indeed, a superficial view of the CMD can lead to the wrong conclusion that variable reddening is present among the stars in NGC 2345, in line with earlier findings from Moffat. However, a careful inspection of the simultaneous position of these stars in the TCD and CMDs is enough to conclude that most of the stars causing the spread are, indeed, foreground stars. The fit of the intrinsic color relation (zero-age main sequence, ZAMS) from Schmidt-Kaler (1982) to the mean cluster sequence in the TCD (upper left panel) provides a reddening $E_{(B-V)}=0.59 \pm 0.04$. Absorption is obviously highly variable across the cluster. This is evident in Figure 4 (upper left panel) where the number of stars decreases strongly to the northwest. However, the effect of variable reddening is not so strong in the cluster area, in agreement with Figure 6 and the low dispersion around the mean color excess. In the CMD (middle upper panel in Figure 6), we show the same ZAMS fitted vertically to the blue envelope of the cluster sequence, which yields an apparent distance modulus $\left(V-M_{V}\right)=14.2 \pm 0.1$. When removing the effects of visual absorption onto the apparent distance modulus, we derive a distance of $3.0 \pm 0.5 \mathrm{kpc}$ from the Sun for NGC 2345. A similar fitting to the $V$ versus $(V-I)$ CMD (right upper panel) confirms previous findings. In both CMDs, an estimate of the age is obtained by superposing the cluster sequence with models from the Padova group (Marigo et al. 2008). The superimposed isochrones bracketing the cluster sequence are for log (age) $=7.8-7.9$ (63-70 Myr).

From our analysis, NGC 2345 turns out to be significantly more distant than the $1.75 \mathrm{kpc}$ given by Moffat (1974). Such a large difference in the cluster distance comes from the fact that Moffat's data only reach $V \sim 14 \mathrm{mag}$, a magnitude level where confusion with field interlopers is particularly high (see Figure 3 in the Moffat paper). At the magnitude cutoff level of the Moffat data, he had no chance to see the run of the lower main sequence (MS) as we instead do in Figure 6. Therefore, our ZAMS fitting is much more robust because it encompasses an almost 6 mag range, down to $V \simeq 18$. 

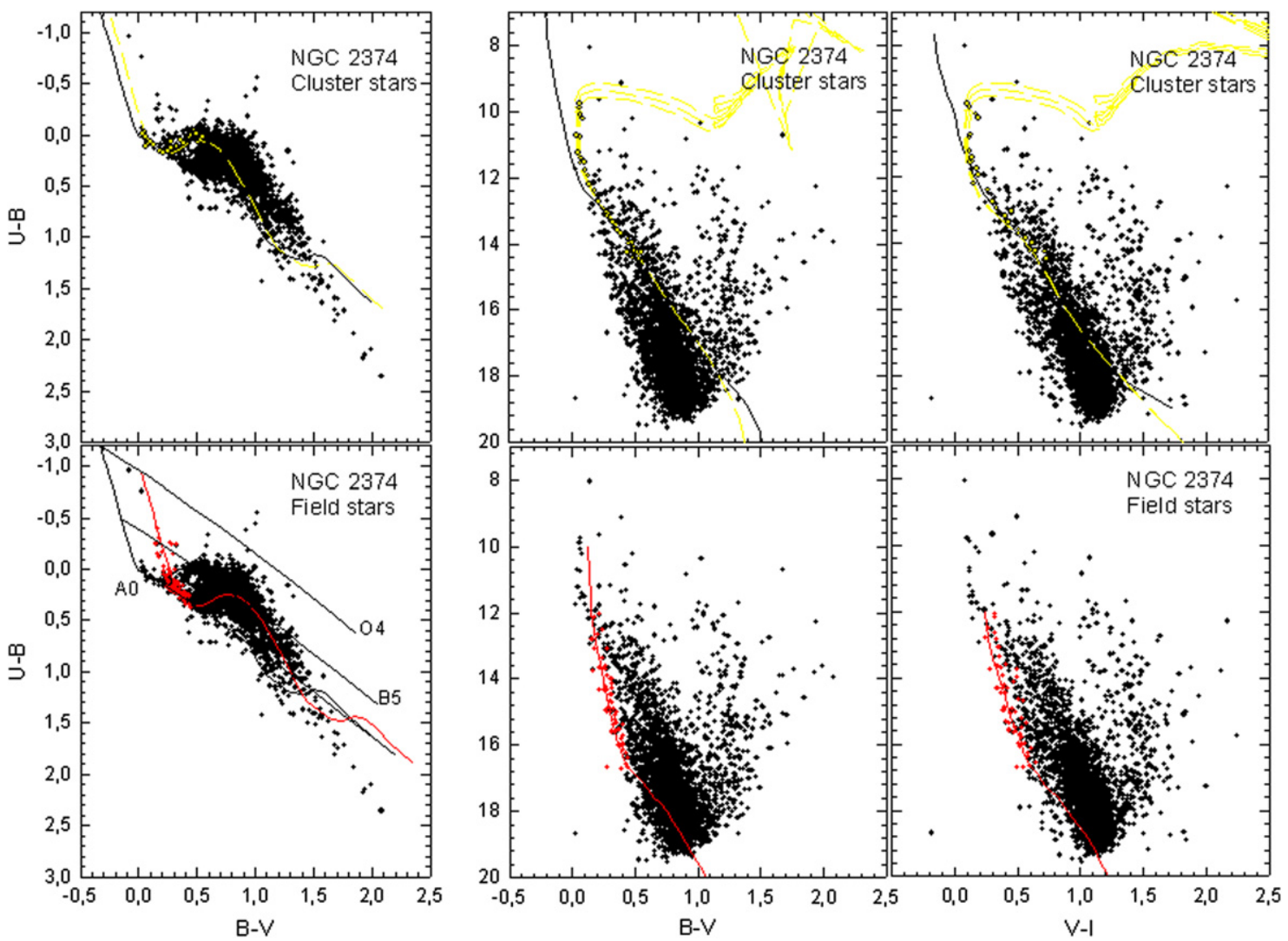

Figure 7. Color-color and color-magnitude diagrams for NGC 2374 (upper panels), and for the surrounding field (lower panels). Yellow dashed lines are isochrones from Marigo et al. (2008) shifted by color excess and distance modulus. The solid line in the two-color diagram is a reddening-free ZAMS, while the dashed yellow line is the same ZAMS shifted by color excess to fit the cluster's probable members (filled yellow symbols). In the lower panels, red colors are used to identify a background young population, and the ZAMS that better fits its sequence. See the text for details.

An interesting final comment is necessary for the sake of clarity and its relevance to the main aim of this work. A close inspection of the lower panels of Figure 6, where supposedly only field stars are present, shows that several stars fall between the reference lines for stars of spectral types O4 and B5. Because these stars have a unique reddening solution in the TCD, we applied the Q method (Straižys 1991) to extract their intrinsic colors, color excesses, and distances, as we have done successfully many times in the past for several other fields (see, e.g., Carraro et al. 2007; Vázquez et al. 2010; Carraro et al 2010a). We will return to these stars again in Section 7.

$N G C$ 2374. In the previous section, we noted that this cluster is not obvious at all from the contour plot in Figure 4 (upper right panel). However, when looking at the upper panels in Figure 7, the presence of a slightly evolved sequence in the CMD is quite evident. The earliest spectral type is $\sim$ B9. This sequence extends for more than five magnitudes in the CMDs and is affected by a uniform reddening $E_{(B-V)}=0.07 \pm 0.03$. Fitting a ZAMS yields an apparent distance modulus of $\left(V-M_{V}\right)=10.90 \pm 0.10$, which, after correcting by visual absorption, places this cluster at a distance of $1.33 \pm 0.25 \mathrm{kpc}$, in agreement with the estimate of Babu (1985). In Figure 7 (upper panels), we have overplotted isochrones from the Padova group (Marigo et al. 2008) on the $V$ versus $(B-V)$ and $V$ versus $(V-I)$ CMDs. They indicate that the cluster is $220-280 \mathrm{Myr}(\log ($ age $)=8.35-8.45)$. This age range indicates that the cluster is older than assumed by Babu (1985), younger than the estimates from Lyngå (1980), $2 \times 10^{9}$ year, and comparable to the age obtained by Fenkart et al. (1972), $3.5 \times 10^{8}$ year.

As we have already mentioned, NGC 2374 is a sparse open cluster and probably it covers a larger area than the one we have surveyed. We adopt as field stars those that do not belong to the cluster sequence we defined in the TCD and CMD upper panels. Inspection of the TCD and CMDs for the stellar field in the direction of NGC 2374 (lower panels in Figure 7) shows the presence of a population of stars (indicated by red symbols) that defines a Blue Plume (BP). In other words, the BP stars do not share the average locus occupied by field stars but they are blueward of the field sequence intersecting it at $V \sim 17-18$ mag.

We remind the reader that previous studies from our group have shown that these stars, the BP stars, do not constitute a physical sequence of bound stars, but a superposition of stars along the line of sight (Carraro et al. 2005). We will return to these stars in the following section.

Haffner 20. This cluster stands out notoriously in the contour plot of Figure 4. Visual inspection of the TCD and CMD (upper panels of Figure 8) for stars within the adopted cluster radius $(\mathrm{r}<2$ arcmin) confirms that Haffner 20 is a physical group. The MS of this cluster is quite clear in the TCD, and is affected by a uniform reddening of $E(B-V)=0.65 \pm 0.03$. Still, like in the case of NGC 2345, contamination by field interlopers is evident in the cluster area, although to a lesser degree. Fitzgerald \& Moffat (1974) give a maximum $E_{(B-V)}$ for this cluster of about 0.55 . Regrettably, they do not show the corresponding TCD of this object (nor the one of Haffner 21), 

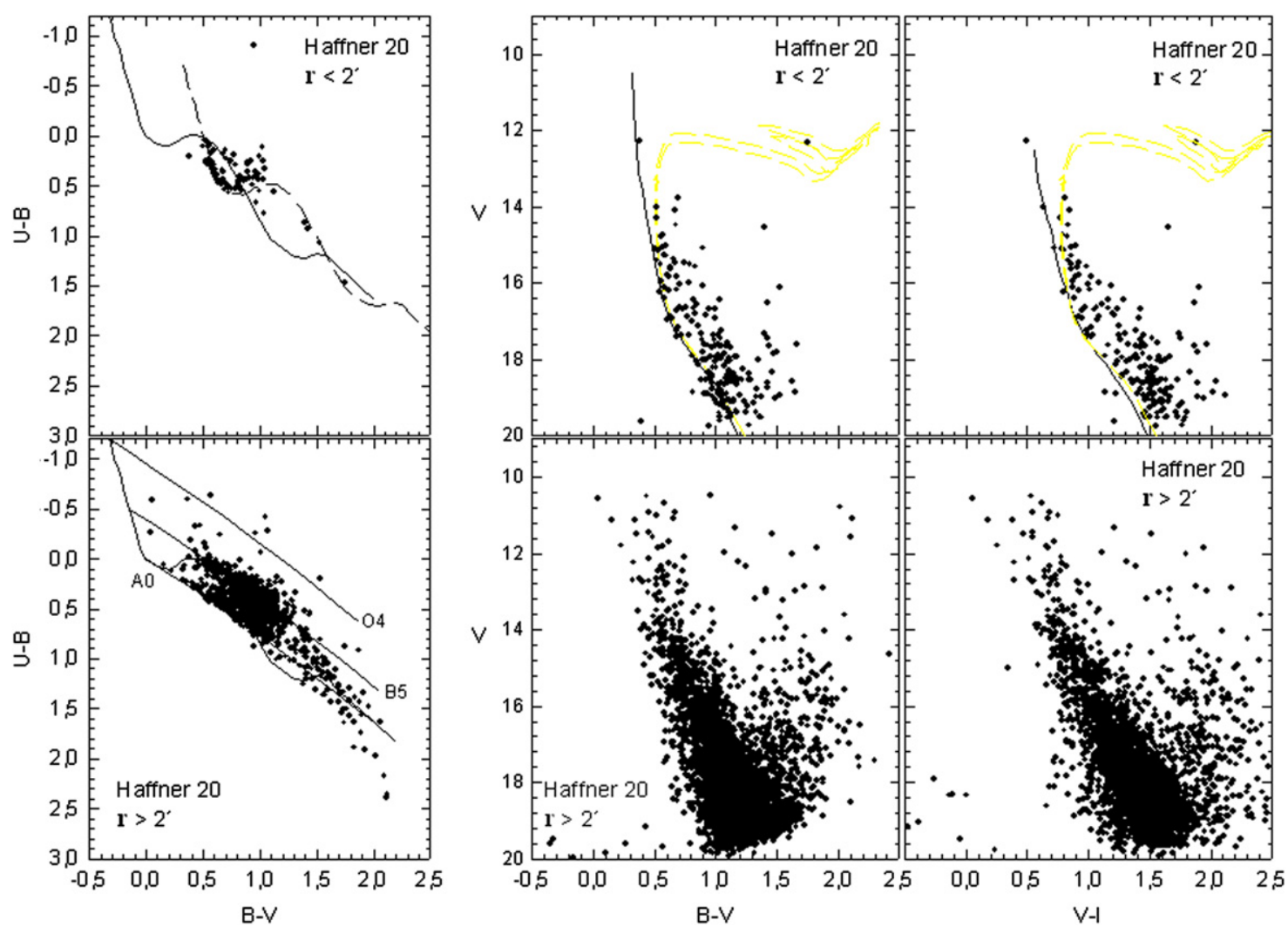

Figure 8. Color-color and color-magnitude diagrams highlighting Haffner 20 members (upper panels), and the surrounding OB field stars (lower panels). Dashed lines are isochrones from Marigo et al. (2008) shifted by color excess and distance modulus. The solid line in the two-color diagram is a reddening-free ZAMS, while the dashed line is the same ZAMS shifted by the color excess to fit the cluster's probable members. Two reference spectral types are indicated in the lower left panel, together with the reddening vector. See the text for details.

but we have redrawn the TCD (not shown to save space) with their data and find a strong data dispersion that makes it impossible to assign a reliable reddening to Haffner 20 (this is fully in line with our photometric comparison in Section 4). The apparent distance modulus $\left(V-M_{V}\right)$ that we infer from ZAMS fitting of the CMD is $15.70 \pm 0.30$, which, in combination with the reddening estimated above, places the cluster at a distance of $5.5 \pm 1.0 \mathrm{kpc}$ from the Sun. This new value is twice that obtained by Fitzgerald \& Moffat (1974) and is the result of the use of a deep photometric data set that allows us to follow the lower cluster MS and perform a more solid ZAMS fitting. The earliest spectral type, as estimated from the TCD, is about B5-B7, which suggests an age of around $100 \mathrm{Myr}$. This is supported by the two isochrones from Marigo et al. (2008) for a $\log (\mathrm{age})$ between 7.95 and 8.00 that we overplotted on the CMDs. This range lowers the cluster age with respect the 200 My suggested by Fitzgerald \& Moffat (1974). The field toward Haffner 20 does not reveal the presence of a young population in the background. There might be various explanations for such an occurrence. One possibility is that the background population is extremely reddened, and we do not detect it because it is confused with the cluster and field MSs. Another possibility is that there are indeed no background populations. Several times in previous works (see, e.g., Carraro et al. 2005) we show cases of the presence and absence of background stellar populations.

Haffner 21. An analysis similar to that of Haffner 20 leads to an estimate of the reddening of $E(B-V)=0.21 \pm 0.03$ (see Figure 9, upper left panel). The MS for the probable members of Haffner 21 is well defined in both the TCD and the CMD, and indicates that Haffner 21 may be slightly older than Haffner 20, because its earliest spectral type would be around B8-B9. From the CMDs (Figure 9, upper right panels) we infer an apparent distance modulus $\left(V-M_{V}\right)=13.35 \pm 0.35$, which puts the cluster at a distance of $3.5 \pm 0.5 \mathrm{kpc}$ from the Sun. In this case, our reddening and distance are in agreement with previous studies from Fitzgerald \& Moffat (1974). The superposition of isochrones from Marigo et al. (2008) indicates that the age range for $\log (\mathrm{age})$ is between 8.05 and 8.1 or 1.1 to $1.25 \times 10^{8}$ years, significantly younger than the $2 \times 10^{8}$ years found by Fitzgerald \& Moffat (1974).

In the control field (lower panels of Figure 9, in red symbols), we detect a young population in the background of the cluster. It is not possible to accurately position this population because it seems to be composed of stars located at very different distances, from close to the Sun to all the way up to about $10 \mathrm{kpc}$ away. We further analyze this population in Section 7.

Trumpler 9. The photometric diagrams for stars within the cluster radius (see Figure 10, upper panels) indicate that we are dealing with an extremely young object. The MS in all the diagrams is very tight, and the reddening solution provides a color excess $E(B-V)=0.20 \pm 0.02$, with no spread. By fitting the CMDs with a ZAMS, we derive an apparent distance modulus $\left(V-M_{V}\right)=12.90 \pm 0.30$. This yields a heliocentric distance of $2.9 \pm 0.5 \mathrm{kpc}$, it larger than the values from Vogt \& Moffat (1972) and Pişmiş (1970). This distance is consistent with Trumpler 9 being part of the Puppis OB1 association, which is also corroborated by our estimate of the cluster age 

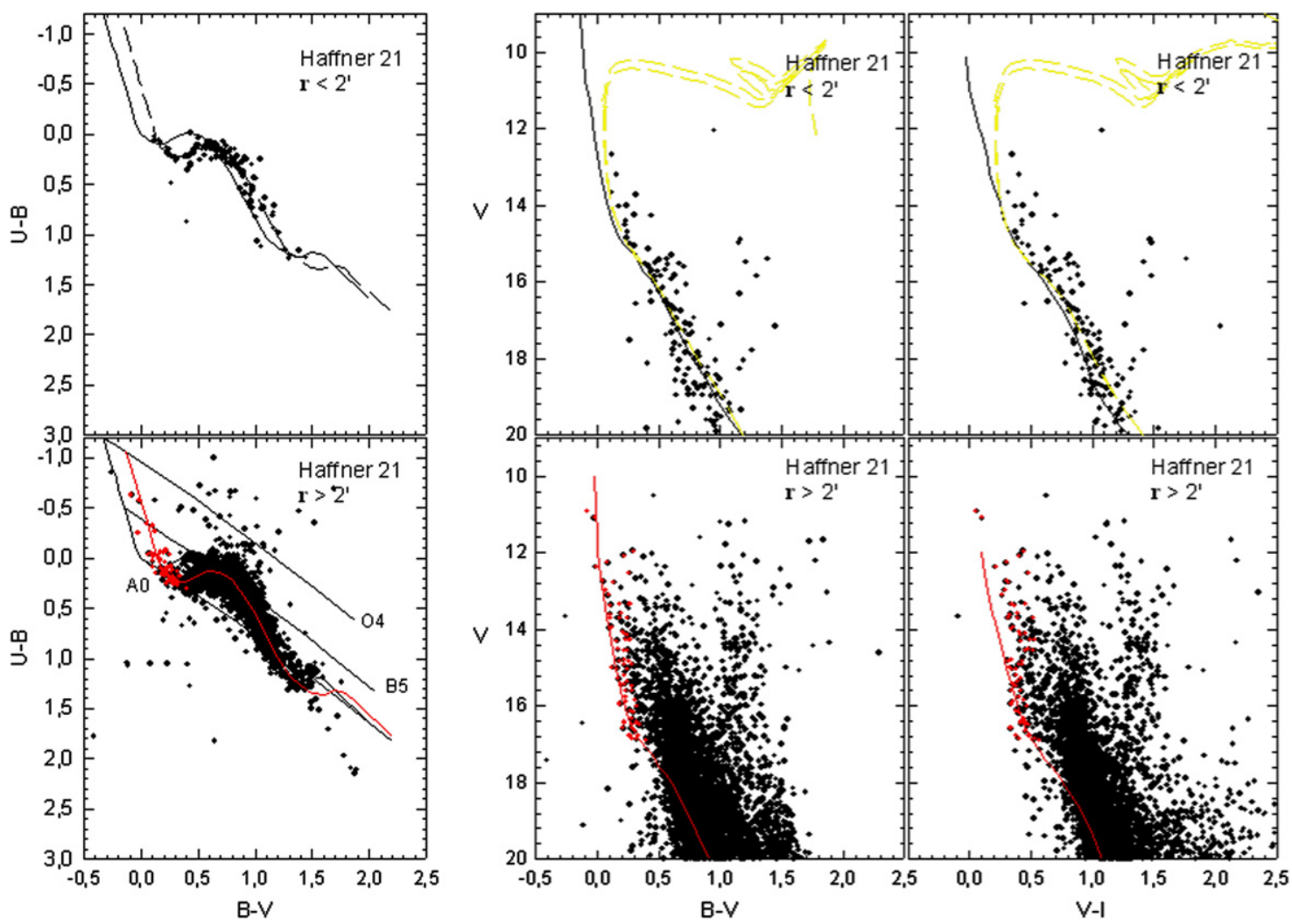

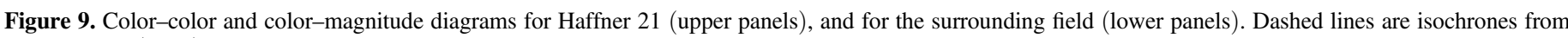

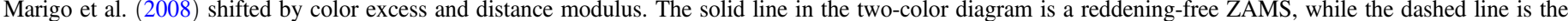

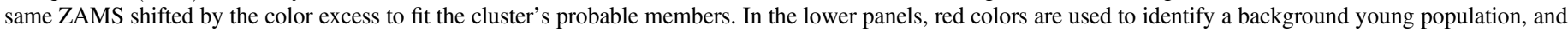
the ZAMS that better fits its sequence. See the text for details.

( $10 \mathrm{Myr})$ from the isochrone fitting. A glance at the lower panels in Figure 10 allows us to conclude that there is also an evident young stellar population in the background of Trumpler 9, as in the case of Haffner 21. Moreover, from the TCD one can conclude that young stars (red symbols) show a wide range of reddening, which conservatively implies that they are located at very different distances, and hence they are tracing an elongated structure in this particular Galactic direction (see below).

\subsection{Trumpler 9, Haffner 20, and Haffner 21 and their Relation to the Puppis Association}

These three clusters are located in a region of the Galaxy historically identified as the Puppis constellation, which contains two prominent $\mathrm{OB}$ associations: Puppis $\mathrm{OB} 1$ and Puppis OB2. The approximate borders of the area are between $l^{\circ} \sim 242$ and $l^{\circ} \sim 246$ and from $b^{\circ} \sim+2$ to $b^{\circ} \sim-1$. Although the nature and properties of these two $\mathrm{OB}$ associations are out of the scope of this work, we would still like to comment on their relationship with Trumpler 9 and Haffner 20 and 21.

We first of all emphasize that no clear understanding of this region is available. Humphreys (1978) found various components of what is designated as the Puppis OB1 association at $2.5 \mathrm{kpc}$ from the Sun, while Havlen (1976) first reported a second, more distant, OB association, Puppis OB2, at $4.3 \mathrm{kpc}$. The existence of these two separate associations has been questioned by Kaltcheva \& Hilditch (2000), who could not find evidence of them but, instead, suggested the presence of two other star groups in the same Galaxy direction (the first at $1 \mathrm{kpc}$, and the second at $3.2 \mathrm{kpc}$ ), but significantly lower, at $b^{\circ} \sim-4$. These would surround the star cluster NGC 2439, located at 3.5-4.5 kpc from the Sun.

Since Trumpler 9 is located at 2.9 and is $10^{6}$ years old, we argue that it is a probable distant member of Puppis OB1. On the other hand, Haffner 20, at $5.5 \mathrm{kpc}$ and about $10^{8}$ years old, would be placed in front of Puppis OB2 and would be an old member of this association. Something similar happens with Haffner 21. Its distance of 3.5 and its comparable age to Haffner 20 would make it a distant member of Puppis OB2.

Finally, let us emphasize that the assessment of these three clusters' membership to these associations (if they really exist) requires an extensive spectroscopic and radial velocity study. In line with the Kaltcheva \& Hilditch (2000) study, the existence of two young stellar groups at $b^{\circ} \sim-4$ and with a distance range of 1-3.2 kpc, in addition to the more distant NGC 2439 open cluster, clearly shows that star formation processes are still vigorous in this region of the Galaxy.

\section{EARLY-TYPE FIELD STARS IN THE FORE- AND BACKGROUND OF THE CLUSTERS}

Groups of evenly distributed early-type stars are frequently found in the background of Galactic open clusters (see, for instance, Carraro et al. 2005) in the third Galactic quadrant. This occurs because Galactic open clusters are mostly located at low Galactic latitudes in this Milky Way region, and the line 

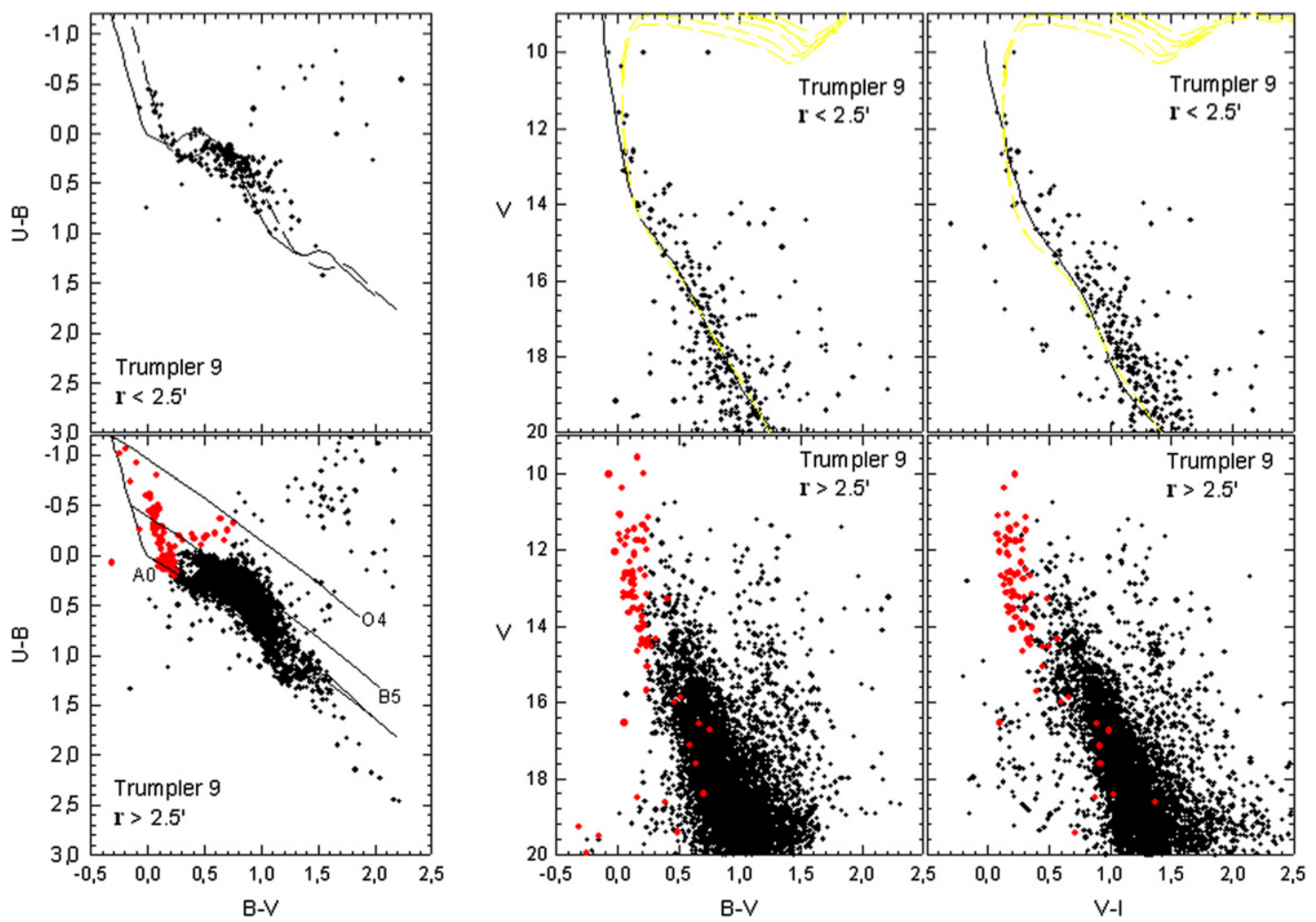

Figure 10. Color-color and color-magnitude diagrams for Trumpler 9 (upper panels), and for the surrounding field (lower panels). Dashed lines are isochrones from Marigo et al. (2008) shifted by color excess and distance modulus. The solid line in the two-color diagram is a reddening-free ZAMS, while the dashed line is the same ZAMS shifted by the color excess to fit the cluster's probable members. In the lower panels, red symbols are used to identify a background young population, and the ZAMS that better fits its sequence. See the text for details.

of sight often intersects parts of distant spiral arms. These stars manifest themselves in classical photometric diagrams in two ways. First, we can spot them in the $U B V$ TCD in the position corresponding to stars for which a unique reddening solution is derived (Straižys 1991). The reddening solution is achieved by moving the stars along the reddening lines back to the position of blue types on the intrinsic (zero reddening) line (see Perren et al. 2012 for a vivid example and a detailed description of the method). Second, in the CMD they tend to define tight sequences, emerging from the left (blue) side of the more prominent MS of the thin (or thick) disk stars. These features are well known as BPs (Carraro et al. 2005; Moitinho et al. 2006), and their exact location in the CMD depends on the line of sight. In this study, field blue stars have been detected in NGC 2345 (Figure 6, lower panels) and Trumpler 9 (Figure 10, lower panels), while BPs are evident in NGC 2374 (Figure 7, lower panels) and Haffner 21 (Figure 9, lower panels). In what follows, we discuss the properties of these groups of stars individually.

\subsection{NGC 2345}

NGC 2345 and NGC 2374 are only $4^{\circ}$ apart on the sky (see Table 1). FNGC 2345 is a young open cluster located at the intersection of the Perseus arm with the LOA. The TCD of stars outside the limits of NGC 2345 (lower panels of Figure 6) shows a large number of stars above the reference line for stars of spectral type B5, with different reddening values roughly along the color range $-0.1 \leqslant(B-V) \leqslant 1.0$. As we mentioned in Section 6, field blue stars in NGC 2345 were de- reddened using the $\mathrm{Q}$ method to derive their intrinsic colors $(B-V)_{0}$ and individual color excesses $E(B-V)$. We remind the reader that once these two quantities are obtained for a star, a spectral type and an absolute magnitude can be assigned using the relationships given in Schmidt-Kaler (1982). This procedure is a variation of the spectroscopic parallax method applied to get individual star distances, which has been proven to be quite solid (see, e.g., Carraro et al. 2007; Carraro et al. 2010a).

In Figure 11, we show the trend of reddening versus distance for the four fields in which blue stars have been detected. To minimize the effects of an erroneous spectral type assignment due to photometric errors, we have only taken into account stars with spectral types earlier than B4. In the case of NGC 2345 , Figure 11 shows the existence of large $E(B-V)$ values, from 0.5 to 1.2 , from the Sun to about $3.5 \mathrm{kpc}$, a distance at which the reddening levels off at a mean value of $E(B-V)=1.2 \pm 0.2$.

Most blue stars in the field of NGC 2345 are found south of the cluster. At a distance of $3.0 \mathrm{kpc}$, the reddening is larger than the reddening of the cluster itself $(E(B-V)=0.59)$, as if the cluster were seen through a dust window. We note that, as shown by the contour plot presented in Figure 4, absorption significantly increases toward the northwest of the cluster.

Finally, from Figure 12 one can note that these early-type stars are found at any distance up to $8 \mathrm{kpc}$, and that a few of them lie at distances larger than $10 \mathrm{kpc}$. All of them are located below the formal $b=0^{\circ}$ plane, roughly following the trend defined by the majority of young open clusters in the first $3 \mathrm{kpc}$ from the Sun. 


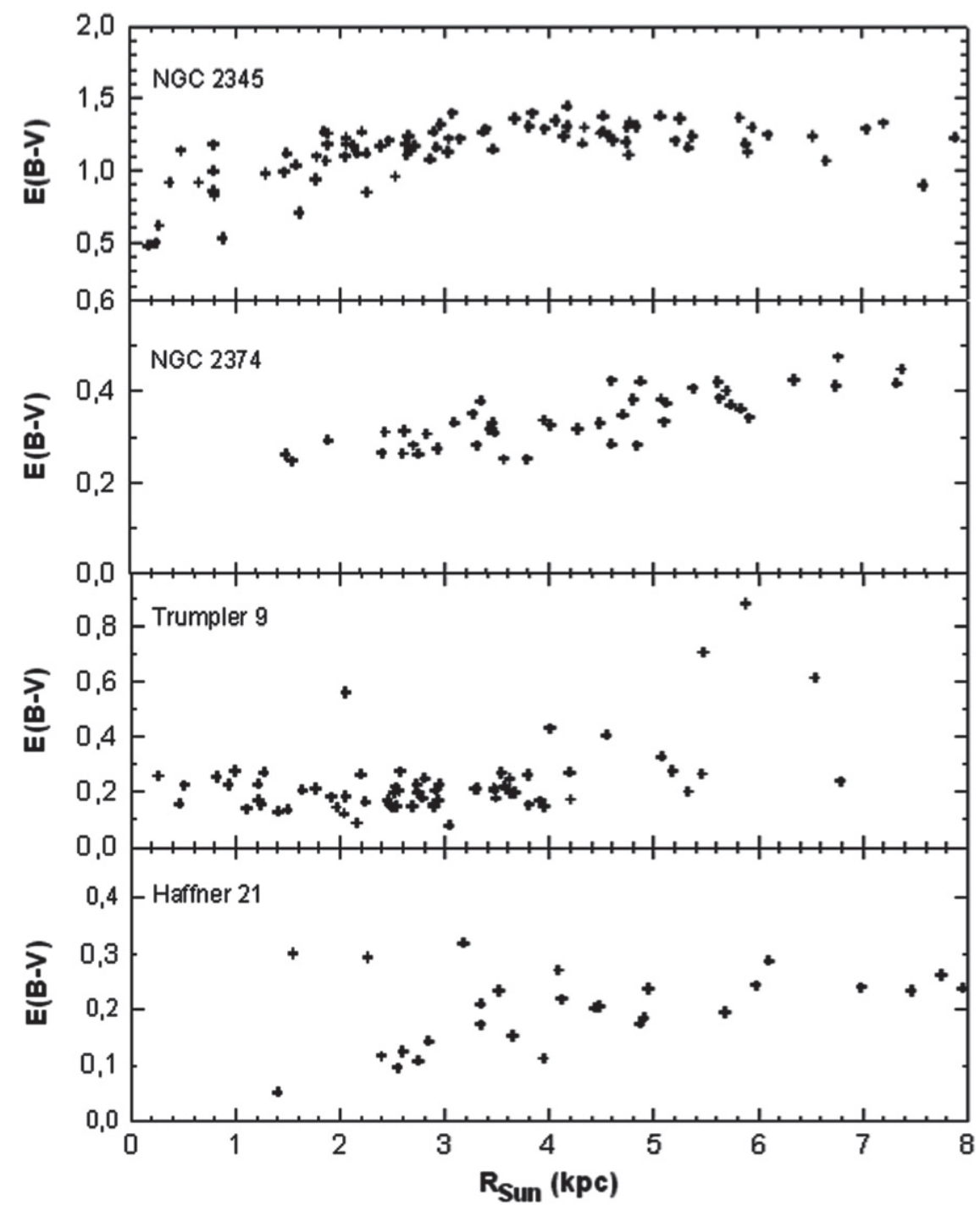

Figure 11. Trend of reddening as a function of distance from the Sun for the five directions studied in this work.

\section{2. $N G C 2374$}

In the CMD of NGC 2374, a clear BP emerges at $V \sim 17$. This BP corresponds to the group of stars with $0.0 \leqslant(B-V) \leqslant 0.5$ in the TCD (lower panels of Figure 7), where we have depicted in red about $50 \mathrm{BP}$ candidate stars. The bulk of these BP stars have a reddening value of $0.40 \pm 0.10$ (Figure 7, left panel) and an apparent distance modulus of $15.2 \pm 0.2$, or a distance of $6.1_{-0.5}^{+0.7} \mathrm{kpc}$ (Figure 7 , middle and right panels).

This BP structure clearly deserves closer attention, so we applied the same method for determining individual distances and color excesses to the $50 \mathrm{BP}$ candidate stars that we used for blue stars in the field of NGC 2345. Inspection of Figure 11 shows in this case that:

1. BP stars begin to appear at $d \sim 1.5 \mathrm{kpc}$ and extend for more than $8 \mathrm{kpc}$,

2. color excess increases steadily from 0.25 to 0.5 , and

3. the earliest photometric spectral type found among the BP stars in NGC 2374 corresponds to B5-type stars, while latest types are around $\mathrm{A} 0$.
At odds with NGC 2374 itself, early-type stars in its background are confined above the formal Galactic disk plane.

\subsection{Haffner 21}

The TCD of field stars in the direction of Haffner 21 (lower left panel of Figure 9) reveals the presence of a strip of stars immediately below the $\mathrm{O} 4$ reddening line, from $(B-V)=0.4$ to 1.2. They are depicted by black symbols.

Haffner 21 exhibits a notorious BP (see Figure 9, lower panel) extending for more than five magnitudes. We marked some of the stars in the BP with red symbols and fitted them with the Schmidt-Kaler ZAMS. This procedure yielded an apparent distance modulus of $15.2 \pm 0.4$ that, for a reddening value of $0.30 \pm 0.12$, corresponds to a distance of $7.1_{-1.2}^{+1.4} \mathrm{kpc}$. We also applied the Q method to obtain the intrinsic properties of these stars, which we assume are representative of the BP. Figure 11 shows that these stars are distributed starting at $1.5 \mathrm{kpc}$ from the Sun, and that the reddening steadily increases up to a value of about 0.25 . 


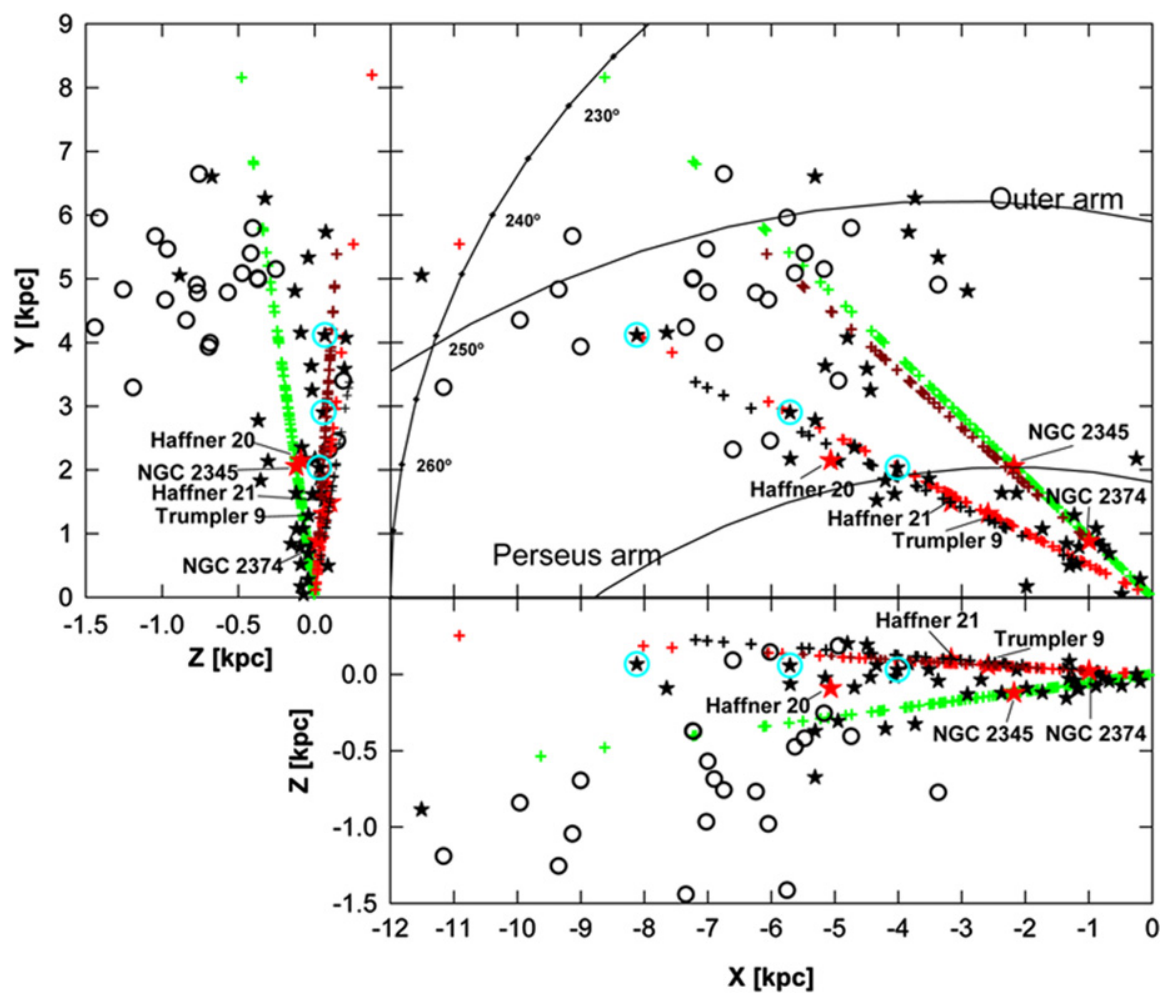

Figure 12. Updated version of Figure 1, where we have added the results of the analysis from the cluster sample presented in this paper. Symbols are as in Figure 1, except that Haffner 18 (1/2) and 19 are highlighted with cyan circles. Red stars indicated the five additional clusters studied in this work. Green, brown, red, and black small crosses correspond to early-type stars in the line of sight to NGC 2345, NGC 2374, Trumpler 9, and Haffner 21, respectively.

\subsection{Trumpler 9}

Trumpler 9 is at 1.3 above the galactic plane and at 1.8 away from Haffner 21; it is also above the galactic plane. These two clusters are located close to the northern edge of the Vela Gum SN remnant. As in the case of NGC 2345, in the field of Trumpler 9 there is an evident group of blue stars outside the cluster's boundary (depicted by red symbols in Figure 10, lower panels). In the TCD they define a strip between the reddening lines for 04- and A0-type stars, thus admitting a unique reddening solution. This situation is reflected in the CMDs (Figure 10, mid and right lower panels), where BP stars are color-coded in red as well. From our analysis, the earliest photometric spectral type found among these stars is about B0.5; Figure 11 shows that their reddening $E(B-V)$ has a mean value of $0.20 \pm 0.10$, which stays constant up to $4 \mathrm{kpc}$. Beyond $4 \mathrm{kpc}$, a few stars show $E(B-V)$ values near 0.9 and helio-distances up to $8 \mathrm{kpc}$. An outlier-having large reddening -is at $2 \mathrm{kpc}$ from the Sun. Both these group are located northeast of Trumpler 9, the most absorbed region covered by our images.

\section{DISCUSSION AND CONCLUSIONS}

In this work we have presented new UBVRI CCD data for five poorly known Galactic star clusters: NGC 2345, NGC 2374, Haffner 20, Haffner 21, and Trumpler 9. Our data supersede previous photographic and photoelectric studies, both in depth and quality, which allowed us to constrain the clusters' basic parameters more solidly. We report in Table 4 the results of this new analysis, in comparison with previous studies.
We stress, however, that the main goal of this work is to study the spatial distribution of early-type field stars that often happen to lie in the background of open clusters in the 3GQ of the Milky Way (Moitinho et al. 2006; Vázquez et al. 2008). To summarize our conclusions along this vein, we refer the reader to Figure 12-which is an updated version of Figure 1, where we have added the new findings that have resulted from the analysis of the cluster sample presented in this paper.

Before interpreting our data, we would like to further stress that our sample is mostly made of bona fide OB stars. In fact, one may wonder whether or not our sample could be significantly contaminated by old hot subdwarf stars, or that the OB stars we detect out of the plane are mainly OB runaway stars. We would like to comment on these two possibilities.

Hot subdwarf stars are often found in the Milky Way. They are associated with old stellar populations, and they are in most cases binaries. The statistics of these stars are poorly known. Early estimates (Downes 1986) count one hot subdwarf every 10 million stars, and one B subdwarf every million stars, when considering one cubic parsec volume. Recent studies (Breedt \& Gansicke 2011 and references therein) confirm these figures. These stars are very faint and, when detected near the Sun, can be confused with distant OB stars in the absence of any spectroscopic information. The typical magnitude of these stars is $\mathrm{M}_{V} \approx+5.0$. This means that with our limiting magnitude of $V \approx 17.5$ for the BPs, we can detect them at a distance modulus up to $(m-M) \approx 12.0$, assuming no reddening. This turns into a distance from the Sun of about $3.0 \mathrm{kpc}$, thus defining a volume of $\sim 340,000$ cubic parsec, since our detectors covers $20 \times 20 \operatorname{arcmin}^{2}$ on the sky. This implies that we expect $0.2 \mathrm{O}$ subdwarfs per cubic parsec, and $0.7 \mathrm{~B}$ subdwarfs per cubic parsec (according to Downes 1986) in each of our pointings. 
Table 4

New and Earlier Results for the Present Cluster Sample

\begin{tabular}{|c|c|c|c|c|c|c|}
\hline Cluster & $\begin{array}{c}\text { Size } \\
\operatorname{arcmin}\end{array}$ & $E_{B-V}$ & $\begin{array}{l}R_{\text {Sun }} \\
\text { kpc }\end{array}$ & $\begin{array}{l}D \\
\mathrm{pc}\end{array}$ & $\begin{array}{c}\text { Age } \\
\left(\times 10^{6}\right) \text { year }\end{array}$ & Reference \\
\hline \multirow[t]{2}{*}{ NGC 2345} & 5.25 & $0.48-1.16$ & 1.75 & $\cdots$ & 60 & Moffat (1974) \\
\hline & 3.75 & 0.59 & 3.00 & 6.6 & $63-70$ & This work \\
\hline \multirow[t]{4}{*}{ NGC 2374} & $\ldots$ & 0.175 & 1.20 & $\ldots$ & 75 & Babu (1985) \\
\hline & $\ldots$ & $\ldots$ & $\ldots$ & $\ldots$ & 2000 & Lyngå (1980) \\
\hline & $\cdots$ & $\cdots$ & $\cdots$ & $\cdots$ & 350 & Fenkart et al. (1972) \\
\hline & $\ldots$ & 0.07 & 1.33 & $\ldots$ & $220-280$ & This work \\
\hline \multirow[t]{3}{*}{ Trumpler 9} & 3.6 & 0.20 & 0.90 & $\cdots$ & $\cdots$ & Pişmiş (1970) \\
\hline & 3.5 & 0.25 & 2.23 & $\cdots$ & $\cdots$ & Vogt \& Moffat (1972) \\
\hline & 2.5 & 0.20 & 2.90 & 4.2 & 10 & This work \\
\hline \multirow[t]{2}{*}{ Haffner 20} & 1.1 & 0.55 & 2.40 & $\cdots$ & 200 & FitzGerald \& Moffat (1974) \\
\hline & $<2.0$ & 0.65 & 5.50 & 6.2 & $90-100$ & This work \\
\hline \multirow[t]{2}{*}{ Haffner 21} & 1.1 & 0.20 & 3.30 & $\cdots$ & 200 & FitzGerald \& Moffat (1974) \\
\hline & $<2.0$ & 0.21 & 3.50 & 4.0 & $110-120$ & This work \\
\hline
\end{tabular}

An additional point is that $\mathrm{OB}$ subdwarf stars do not define the typical tilted BP sequences we have found, which, on the other hand, resemble more the MS of nearby young open clusters.

OB runaway stars are also often found in the Milky Way disk. They are typically associated with massive young open clusters (e.g., Westerlund 2, Carraro et al. 2013; Roman-Lopes et al. 2011), from which they escaped (Fujii \& Portegies Zwart 2011). First of all, we stress that the escaping direction is not always vertical with respect to the disk plane, but it is clearly random. In Westerlund 2, we believe there are two runaway $\mathrm{OB}$ stars out of about $50 \mathrm{OB}$ stars in the cluster. No $\mathrm{OB}$ runaway stars have been reported in young open clusters in the anti-center direction, most likely because clusters are not massive enough to produce this phenomenon (Fujii \& Portegies Zwart 2011), which indeed is mostly seen in the nearby massive star cluster located within the solar ring. Even assuming that the proportion of runaway stars in the outer disk is the same as in the inner, say about $20 \%$, it is difficult to reproduce the high number of off-plane OB stars we are finding.

In Figure 12, which covers the whole 3GQ, from $180^{\circ}$ to $270^{\circ}$ in Galactic longitude, we depict the positions of the young open clusters studied by our group, and of overdensites of young stars that we have discovered in their background or foreground. In this figure, the Sun is located at $(0,0,0)$. Black stars are young open clusters from our previous studies (Moitinho et al. 2006; and Vázquez et al. 2008, 2010), the two black stars surrounded by a blue circle are Haffner 18 and Haffner 19, and the five red stars are the clusters analyzed in this work. Crosses mark the distribution of early-type stars in the directions of NGC 2345 (green), NGC 2374 (brown), Trumpler 9 (red), and Haffner 21 (black), respectively. Open circles are BPs detected in previous studies. The solid logarithmic curves are the approximate extrapolated locations of the Perseus and Outer arms, according to the Valleé (2008) model.

The addition of the five clusters studied here has added a wealth of new information on the structure of the Galactic thin disk in the $3 \mathrm{GQ}$, thanks to the fact that four of them (the exception being Haffner 20) lie in fields rich in early-type stars located at very different distances from the Sun.

NGC 2345 and the associated blue stars follow the wellestablished structure of the disk in the 3GQ: the disk keeps close the formal $b=0^{\circ}$ Galactic plane up to $\sim 4 \mathrm{kpc}$ from the
Sun, and then starts to bend down following the Galactic warp. However, field stars in the direction of NGC 2374, Trumpler 9, and Haffner 21 are found all the way up to the location of the outer arm, and, these clusters being at positive Galactic latitudes, they define distributions of young stars that, at odds with any previous expectations, lie above or close to the formal $b=0^{\circ}$ Galactic plane. In other words, as illustrated in the lower panel in Figure 12, they do not follow the Galactic warp. Beyond $\sim 4 \mathrm{kpc}$ from the Sun, the Galactic thin disk, as defined by young stars and open clusters, thickens significantly, and this thickening keeps increasing because of the sudden appearance of the Galactic warp.

We interpret this situation as an indication that the Galactic thin disk is not only warped, but also flared, like the thick disk (Momany et al. 2006; López-Corredoira \& Molgó 2014). In other words, the Galactic thin disk scale height seems to increase moving outward from the Sun. This interpretation nicely explains the unusual, unexpected location of Haffner 18 and Haffner 19 (see Figure 12): they are very young (NGC 2374, Trumpler 9, and Haffner 21) and distant, but lie far from the main-warped-thin disk location. The addition of background blue stars associated with three more clusterssharing the same position as Haffner 18 and 19-lends further support to this scenario.

One may wonder whether other independent observational evidence of this thickening of the disk is available, in addition to the few stellar fields discussed here. We note that in their recent all-sky survey of HiI regions in the Wide-Field Infrared Survey Explorer database, Anderson et al. (2014) detected quite a large number of HII regions in the Galactic anti-center. Their Figure 4 shows very clearly both the warp and the flare of the thin disk in the second and third Galactic quadrants $\left(90^{\circ} \leqslant l \leqslant 270^{\circ}\right)$, nicely confirming our findings.

To conclude, we have shown in our series of papers, including this one, that early-type stars and very young open clusters not only follow the warp in the 3GQ, but they also contribute to the flaring of the thin disk beyond $\sim 4 \mathrm{kpc}$ from the Sun. This might imply that in the outer disk spiral arms are thicker than commonly believed. In fact a large fraction of the stars we have studied are younger than $100 \mathrm{Myr}$; hence, it is conceivable to think that they are not displaced much from their birthplaces. This implies that these young stars formed out of vertically dispersed material, which is not concentrated into the 
plane but can be found even at large distances from the mean plane.

This interpretation is supported by evidence (Carraro et al. 2005; Vázquez et al. 2008) that, besides early-type stars, CO clouds are also found at any vertical distance in the 3GQ quadrant, and their spatial distribution closely follows young stars and star clusters. In a very recent study, Suad et al. (2014) performed a search of Hi super-shells in the second and third quadrant of the Galaxy showing that Hi super-shells can be found up to $3 \mathrm{kpc}$ above the galactic plane $(50 \%$ of them are at less than $500 \mathrm{pc}$ above the galactic plane). Early-type stars, young clusters, $\mathrm{CO}$ clouds, and $\mathrm{HI}_{\mathrm{i}}$ super-shells all show the same smoothed distribution that thickens the outer Galactic disk.

G.C. thanks Yazan Momany for many fruitful discussions. R.A.V. and J.A.A. acknowledge the ESO for granting a visitorship at the ESO premises in Santiago, where part of this work was done. E.C. acknowledges support by the Fondo Nacional de Investigación Científica y Tecnológica (Project No. 1110100 Fondecyt), and the Chilean Centro de Excelencia en Astrofísica y Tecnologías Afines (PFB06). R.A.V. and E.E. G. acknowledge financial support from the PIP 1359 from CONICET. This research used the WEBDA database, maintained at the Institute for Astronomy of the University of Vienna. Finally, we greatly appreciate the input of the referee.

\section{REFERENCES}

Aarseth, S. J. 1996, in ASP Conf. Ser. 90, The Origins, Evolution, and Destinies of Binary Stars in Clusters, ed. E. F. Milone, \& J.-C. Mermilliod (San Francisco, CA: ASP) 423

Alfaro, E. J., Cabrera-Cano, J., \& Delgado, A. J. 1991, ApJ, 378, 106 Anderson, L. D., Bania, T. M., Balser, D. S., et al. 2014, ApJS, 212, 1 Babu, S. 1985, JApA, 6, 61

Baume, G., Moitinho, A., Giorgi, E. E., Carraro, G., \& Vázquez, R. A. 2004, A\&A, 417, 961

Breedt, E., \& Gansicke, B. T. 2011, in ASP Conf. Ser. 447, Evolution of Compact Binaries, ed. L. Schmidtobreick, M. R. Schreiber, \& C. Tappert (San Francisco, CA: ASP), 203

Carraro, G. 2011, A\&A, 536, 101

Carraro, G. 2014, in Proc. IAU Symp. 298, Setting the Scene for Gaia and LAMOST, ed. S. Feltzing, G. Zhao, N. A. Walton, \& P. A. Whitelock (Cambridge: Cambridge Univ. Press), 7
Carraro, G., Costa, E., \& Ahumada, J. A. 2010b, AJ, 140, 954

Carraro, G., Vázquez, R. A., Costa, E., Perren, G., \& Moitinho, A. 2010a, ApJ, 718,683

Carraro, G., Moitinho, A., Zoccali, M., Vázquez, R. A., \& Baume, G. 2007, AJ, 133, 1058

Carraro, G., Perren, G., Vázquez, R. A., \& Moitinho, A. 2014, in ASP Conf. Ser. 480, Structure and Dynamics of Disk Galaxies, ed. M. Seigar, \& P. Treuthardt (San Francisco, CA: ASP), 10

Carraro, G., Vázquez, R. A., Moitinho, A., \& Baume, G. 2005, ApJL, 630, L153

Carraro, G., Turner, D., Majaess, D., \& Baume, G. 2013, A\&A, 555, 50

de La Fuente Marcos, R. 1997, A\&A, 322, 764

Downes, R. A. 1986, ApJS, 61, 569

Fenkart, R. P., Buser, R., Ritter, H., et al. 1972, A\&AS, 7, 48

Fitzgerald, M. P., \& Moffat, A. F. J. 1974, PASP, 86, 480

Fujii, M. S., \& Portegies Zwart, S. 2011, Sci, 334, 1380

Havlen, R. J. 1976, A\&A, 47, 193

Humphreys, R. M. 1978, ApJS, 38, 309

Kaltcheva, N. T., \& Hilditch, R. W. 2000, MNRAS, 312, 753

King, I. 1962, AJ, 67, 471

Kroupa, P., Aarseth, S., \& Hurley, J. 2001, MNRAS, 321, 699

Landolt, A. U. 1992, AJ, 104, 372

Levine, E. S., Blitz, L., \& Heiles, C. 2006, Sci, 312, 1773

Lyngå, G. 1980, A Computer Readable Catalogue of Open Cluster Data (France: Stellar Data Centre, Observatoire de Strasbourg)

López-Corredoira, M., \& Molgó, J. 2014, A\&A, 567, 106

Marigo, P., Girardi, L., Bressan, A., et al. 2008, A\&A, 482, 883

Martin, N., Ibata, R. A., Bellazzini, M., et al. 2004, MNRAS, 348, 12

Moffat, A. F. M. 1974, A\&AS, 16, 33

Moitinho, A. 2001, A\&A, 370, 436

Moitinho, A., Vázquez, R. A., Carraro, G., et al. 2006, MNRAS, 368, L77

Momany, Y., Zaggia, S., Gilmore, G., et al. 2006, A\&A, 451, 515

Perren, G., Vázquez, R. A., \& Carraro, G. 2012, A\&A, 548, 125

Pişmiş, P. 1970, BOTT, 5, 293

Robin, A., Creze, M., \& Mohan, V. 1992, ApJL, 400, L25

Roman-Lopes, A., Barba, R. H., \& Morrell, N. I. 2011, MNRAS, 416, 501

Schmidt-Kaler, Th. 1982, in Landolt-Börnstein, Numerical data and Functional Relationships in Science and Technology-New Series, Vol. 2(b), ed. K. Schaifers, \& H. H. Voigt (Berlín: Springer), 14

Skrutskie, M. F., Cutri, R. M., Stiening, R., et al. 2006, AJ, 131, 1163

Stetson, P. B. 1987, PASP, 99, 191

Straižys, V. 1991, in Multicolor Photometry, Astronomy and Astrophysics Series, Vol. 15 (Tucson, AZ: Pachart Publishing House)

Suad, L. A., Caiafa, C. F., Arnal, W. M., \& Cichowolski, S. 2014, A\&A, 564,116

Vallée, J. P. 2008, A\&J, 135, 1301

Vázquez, R. A., May, J., Carraro, G., et al. 2008, ApJ, 672, 930

Vázquez, R. A., Moitinho, A., Carraro, G., \& Dias, W. S. 2010, A\&A, 511, 38

Vogt, N., \& Moffat, A. F. J. 1972, A\&AS, 7, 133 